Article

\title{
Decomposition and Arrow-Like Aggregation of Fuzzy Preferences
}

\author{
Armajac Raventós-Pujol ${ }^{1}$, María J. Campión ${ }^{1}$ () and Esteban Induráin ${ }^{2, *}$ (D) \\ 1 Institute for Advanced Research in Business and Economics and Departamento de Estadística, Informática y \\ Matemáticas, Universidad Pública de Navarra, 31006 Pamplona, Spain; \\ armajac.raventos@unavarra.es (A.R.-P.); mjesus.campion@unavarra.es (M.J.C.) \\ 2 Institute for Advanced Materials and Departamento de Estadística, Informática y Matemáticas, Universidad \\ Pública de Navarra, 31006 Pamplona, Spain \\ * Correspondence: steiner@unavarra.es; Tel.: +34-948169551
}

Received: 4 February 2020; Accepted: 12 March 2020; Published: 17 March 2020

check for updates

\begin{abstract}
We analyze the concept of a fuzzy preference on a set of alternatives, and how it can be decomposed in a triplet of new fuzzy binary relations that represent strict preference, weak preference and indifference. In this setting, we analyze the problem of aggregation of individual fuzzy preferences in a society into a global one that represents the whole society and accomplishes a shortlist of common-sense properties in the spirit of the Arrovian model for crisp preferences. We introduce a new technique that allows us to control a fuzzy preference by means of five crisp binary relations. This leads to an Arrovian impossibility theorem in this particular fuzzy setting.
\end{abstract}

Keywords: Arrow's impossibility theorems; mathematical social choice; fuzzy preferences; decomposition of preferences; aggregation of individual profiles; social rules; Arrovian models, Paretian property; independence of irrelevant alternatives; dictatorship

MSC: 62C86 (Primary); 03E76; 91B14; $91 \mathrm{B06}$ (Secondary)

\section{Introduction}

The famous Arrow's impossibility theorem encountered in Social Choice (see e.g., [1-6]) states that under a mild set of restrictions of common sense, the preferences, defined on a universe $U$ of at least three alternatives, of the individual members of a finite society $N$ of at last three individuals, cannot be aggregated into a new social preference. This impossibility result appears in the crisp setting so that the preferences of the individuals are total preorders on $U$. Each preference is actually a binary relation (denote it by $\mathcal{R}$ ) understood as a subset of the Cartesian product $U \times U$. This subset is crisp, that is, its membership function $\mu$ takes values in the set $\{0,1\}$, so that when $\mu(x, y)=1$ we interpret that $x$ is related to $y$ through $\mathcal{R}$-we denote it by $x \mathcal{R} y$-or, formally and equivalently $(x, y) \in \mathcal{R} \subseteq U \times U$. Obviously, when $\mu(x, y)=0$ we would interpret that $x$ is not preferred to $y$.

If instead of considering crisp binary relations on the set of alternatives $U$, we deal with graded membership functions that may take any possible value in the unit interval $[0,1]$, so that the corresponding relations now become fuzzy subsets of the Cartesian product $U \times U$, unlike the crisp approach, it may happen in several contexts that some Arrow-like aggregation of fuzzy preferences is still possible (see e.g., [7,8]). In other words, passing to a fuzzy context gives us the opportunity of looking for good aggregation rules à la Arrow, after all. In fact, there are many possible generalizations of the Arrovian model to the fuzzy approach.

Most Arrow-like models in the fuzzy setting pay attention to the possible generalizations of the restrictions involved in the model and imposed to the aggregation rules (see e.g., [8], where four 
different extensions of the so-called condition of independence of irrelevant alternatives have been launched).

However, the generalizations to the fuzzy set of the key concept of a preference seem to have been somewhat disregarded. In the present paper, first, we would like to focus on this point, with the aim of achieving a correct understanding of what a fuzzy preference should be.

Perhaps surprisingly, some of the fuzzy Arrovian models encountered in the specialized literature give rise to possibility theorems, whereas others still generate impossibility results (see e.g., [8,9]). In this direction, the impact of fuzziness in the consideration and handling of this kind of social choice paradoxes has been already discussed in [10] (see also [11-15]).

One of the main reasons for that impact is, obviously, the fact that, unlike crisp preferences, sometimes in a particular Arrovian fuzzy contexts it is possible to aggregate fuzzy preferences into a non-dictatorial one (see e.g., [7]). This is understood as a possibility result. In addition, when a possibility result of that kind appears, one may think that crisp individual preferences, considered as particular cases of fuzzy preferences can actually be fused into a social one. At this stage it is crucial to take into account that the result of that aggregation is, a fortiori, non-crisp. This is a direct consequence of Arrow's impossibility theorem for the crisp setting. In other words, when a possibility result appears for fuzzy preferences, we may expect non-crisp preferences as the result of the fusion. To put an example, in the Arrovian model considered in [7], Proposition 9, the rank of the resulting preferences is $\left\{\frac{1}{2}, 1\right\}$. Even being dychotomic, they do not take the value 0 , so they are not crisp.

In other fuzzy models, as in the considered in the present manuscript, the fuzzy preferences could still be controlled by a finite set of crisp binary relations. So that if crisp binary relations of that kind cannot be fused in an Arrovian setting, the corresponding fuzzy model gives rise to an impossibility theorem à la Arrow. This is a key idea that generates a new technique, introduced throughout this paper. This technique allows us to show that for certain fuzzy preferences, a generalization of the Arrow's impossibility theorem arises, and no social rule exists satisfying all the restrictions of the corresponding extended Arrovian model.

As mentioned above, some impossibility results were already known in particular Arrovian fuzzy approaches (see e.g., [16-22].

Therefore, our aim is not only to prove one more impossibility result, but, instead, introduce a bridge between the fuzzy approach and the crisp setting, interpreting fuzzy preferences by means of five crisp binary relations through the key concept of a fuzzy pseudo-fuzzy preference. As a by-product, this new technique allows us to prove an impossibility result.

The structure of the paper goes as follows:

After the introduction and the subsequent section of preliminaries, we focus on the concept of a decomposition of a fuzzy binary relation. We study, in particular, the uniqueness of decompositions. Then we define fuzzy preferences. Through suitable decompositions, a fuzzy preference will be understood as a triplet $(P, R, I)$ of fuzzy binary relations on a set of alternatives, such that $P$ (respectively, $R$ and $I$ ) plays the role of a strict preference (respectively, of a weak preference, of an indifference). Then we study situations arising in the social choice context in which individual fuzzy preferences need to be fused into a social one. Obviously, questions related to aggregation of fuzzy preferences appear now in a natural way. If the aggregation rules should accomplish some restrictions imposed a priori, we will have fuzzy social choice models. Among them we will analyze here some extensions of the (crisp) Arrovian model. We introduce the concept of a fuzzy pseudo-fuzzy preference that allows us to control fuzzy preferences by means of a finite set of crisp binary relations. Finally, in the fuzzy approach, an impossibility result appears related to this new concept and the subsequent technique introduced here. 


\section{Preliminaries}

\subsection{Preorders on a Set}

Let $X$ stand for a nonempty abstract set.

Definition 1. A binary relation $\mathcal{R}$ on $X$ is a subset of the Cartesian product $X \times X$. Given $x, y \in X$, $(x, y) \in \mathcal{R} \subseteq X \times X$ is also denoted as $x \mathcal{R} y$.

A preorder $\succsim$ on the set $X$ is a reflexive and transitive binary relation on $X$. A preorder $\succsim$ on $X$ is said to be total or complete if $x \mathcal{R} y$ or $y \mathcal{R} y$ holds true for every $x, y \in X$. Given a preorder $\succsim$ on $X$, its asymmetric part, denoted by $\succ$, is the binary relation defined by $x \succ y \Leftrightarrow(x \succsim y) \wedge \neg(y \succsim x)(x, y \in X)$. (Here " $\neg$ " is the standard notation for negation). The symmetric part of $\succsim$, denoted by $\sim$, is the binary relation defined by $x \sim y \Leftrightarrow(x \succsim y) \wedge(y \succsim x)(x, y \in X)$.

Definition 2. Let $\mathcal{R}, \mathcal{R}_{1}, \ldots, \mathcal{R}_{n}$ be $(n+1)$ binary relations on $X$. We say that $\mathcal{R}$ decomposes into $\left\{\mathcal{R}_{1}, \ldots, \mathcal{R}_{n}\right\}$, or, equivalently, $\left\{\mathcal{R}_{1}, \ldots, \mathcal{R}_{n}\right\}$ is a decomposition of $\mathcal{R}$ if $\mathcal{R}=\bigcup_{i=1}^{n} \mathcal{R}_{i}$, and $\mathcal{R}_{i} \cap \mathcal{R}_{j}=\varnothing$ holds true for every $i \neq j \in\{1, \ldots, n\}$. Here $\mathcal{R}_{1}, \ldots, \mathcal{R}_{n}$ are said to be the components of $\mathcal{R}$.

Theorem 1. Let $\succsim$ be a preorder on a set X. The preorder $\succsim$ decomposes into $\succ$ and $\sim$. Moreover, $\{\succ, \sim\}$ is the unique decomposition of $\succsim$ into an asymmetric and a symmetric binary relations. (A binary relation $\mathcal{R}$ on a nonempty set $X$ is symmetric if $x \mathcal{R} y \Rightarrow y \mathcal{R} x$ holds true for every $x, y \in X$, and it is asymmetric if $x \mathcal{R} y \Rightarrow \neg(y \mathcal{R} x)$ holds true for every $x, y \in X)$.

Proof. It is straightforward to see that $\succsim$ decomposes into $\succ$ and $\sim$. By their own definitions it is clear that $\succ$ is asymmetric and $\sim$ is symmetric. Let us see now that the decomposition $\{\succ, \sim\}$ is indeed unique. To prove this fact, suppose that $\succsim$ decomposes into two relations $R_{a}$ and $R_{s}$ with $R_{a}$ asymmetric and $R_{s}$ symmetric. First we will prove that $\succ$ agrees with $R_{a}$.

Suppose that $x \succ y$ but $x R_{a} y$ does not hold, then since $x \succsim y$ and $\succsim$ decomposes as $R_{a} \cup R_{S}$, it follows that $x R_{s} y$. Since $R_{s}$ is symmetric, we also get $y R_{s} x$, which implies that $y \succsim x$, a contradiction. Hence $\succ \subseteq R_{a}$.

Assume now that $x R_{a} y$ but not $x \succ y$. By a similar argument we get that $x \sim y$, because $(x, y) \in \succsim$ and this binary relation decomposes in $\succ \cup \sim$. By asymmetry of $R_{a}$ we actually have that $y R_{a} x$ does not hold. However, $y \sim x$ implies $y \succsim x$, so we get that $y R_{s} x$. Finally, since $R_{s}$ is symmetric, it follows that $(x, y) \in R_{a} \cap R_{s}$. This is a contradiction because, by hypothesis and definition of a decomposition, this intersection is empty. So $R_{a} \subseteq \succ$, and $\succ$ is $R_{a}$.

If $R_{a}$ is $\succ$ it is then clear that $R_{s}$ is $\sim$. This finishes the proof.

\subsection{The Arrovian Model in Social Choice}

Definition 3. Henceforward, unless otherwise stated, a preference over a set X will be understood as a total preorder defined on $X$. Given a preference $\succsim$ on $X$ and $x, y \in X, x \succsim y$ will be interpreted as " $x$ is weakly preferred over $y$ ", or, roughly speaking " $x$ is at least as good as $y$ ". Similarly, $x \succ y$ will mean " $x$ is strictly preferred over $y$ ". Finally, $x \sim y$ will be interpreted as " $x$ and $y$ are indifferent". The binary relation $\succsim$ is said to be the weak preference relation, whereas $\succ$ is the strict preference relation and $\sim$ the indifference relation.

A society is a finite (nonempty) set of individuals. We will denote it by N. For convenience, we will suppose that $N=\{1, \ldots, n\}$. The elements or individuals of the society $N$ will define their preferences over a nonempty set $X$. This set $X$ is usually known as the set of alternatives. Unlike $N$, which has been defined as being finite, the cardinality of the set $X$ is not restricted. So X could be infinite. The set of all total preorders on $X$ is denoted by $\mathcal{O}_{X}$.

A profile of the $n$ individuals of a society $N$ is an $n$-tuple $\left(\succsim_{1}, \ldots, \succsim_{n}\right)$ of preferences defined on a set $X$ of alternatives. Here, $\succsim_{i}$ is the preference of the individual $i \in N$. For economy of language $\left(\succsim_{1}, \ldots, \succsim_{n}\right)$ can be abbreviated by $\left(\succsim_{i}\right)$. 
A choice function on a set $X$ is a map $c$ from the power set of $X$ into itself satisfying that $c(v) \subseteq v$, for every $v \in \mathcal{P}(X)$. These sets $v$ are called agendas. (The power set of a set $X$ is the collection of all the subsets of X. We will denote it by $\mathcal{P}(X)$. Another classical notation is $2^{X}$ ).

Definition 4. Let $X$ be a nonempty set and $n \in \mathbb{N}$ a strictly positive natural number. A social choice rule on $X$ is a map from a set $D \subseteq \mathcal{O}_{X}^{n}$ of profiles of $n$ individuals to the set of choice functions on $X$. Once a social choice rule is fixed, the image of a a profile $\left(\succsim_{i}\right)$ is denoted by $c_{\left(\succsim_{i}\right)}$.

At this point, we can think about which properties must have the choice rules in order to be considered as fair. In the following definitions, we will introduce some properties which have been usually requested to social choice rules, bearing in mind an idea of "common sense". These concepts were already introduced by renowned researchers in the first half of the twentieth century. In fact, we will mainly focus on the properties coming from the so-called Arrovian model, introduced by Kenneth J. Arrow in the 1950's (see [1,2]). The underlying idea of these definitions is to establish a suitable system of axioms to deal with good social choice rules.

Definition 5. A social choice rule on a set $X$ is said to satisfy the property of universal domain if it is defined on the whole set $\mathcal{O}_{X}^{n}$.

It is reasonable to think that if all individuals in a society do actually prefer an alternative $x$ over another $y$, it would be really strange if $y$ were socially selected but $x$ were not chosen. The next definition formalizes this idea.

Definition 6. A social choice rule c on a set X of alternatives is said to be Paretian if for every pair of alternatives $x, y \in X$ and every profile $\left(\succsim_{i}\right)$ on the domain of $c$, it holds true that if $x \succsim_{i} y$ for any individual $i \in N$ and there exist an individual $j \in N$ with $x \succ_{j} y$, then for every agenda $v \in \mathcal{P}(X)$ we have that if $x \in v$, then $y \notin c_{\left(\succsim_{i}\right)}(v)$.

The next property reflects the idea that the choice restricted to a subset of alternatives only depends on the individual preferences over that given subset.

Definition 7. A social choice rule $c$ on $X$ satisfies the condition of independence of irrelevant alternatives if for every agenda $v \in \mathcal{P}(X)$ and every pair of profiles $\left(\succsim_{i}\right)$ and $\left(\succsim_{i}^{\prime}\right)$ on the domain of $c$, such that the restrictions of $\succsim_{i}$ and $\succsim_{i}^{\prime}$ to $v$ coincide for all $i \in N$, it holds true that $c_{\left(\succsim_{i}\right)}(v)=c_{\left(\succsim_{i}^{\prime}\right)}(v)$.

The next property is devoted to avoiding the existence of a single individual in the society whose decision power over the final output of social choice is absolute. First, we introduce the concept of a dictator.

Definition 8. A social choice rule $c$ on $X$ is said to be dictatorial if there exists a $k \in N$, known as the dictator, such that for every $x, y \in X$, every profile $\left(\succsim_{i}\right)$ with $x \succ_{k} y$ and any agenda $v \in \mathcal{P}(X)$, it holds true that if $x \in v$ then $y \notin c_{\left(\succsim_{i}\right)}(v)$. Accordingly, the rule $c$ is said to satisfy the condition of non-dictatorship if it fails to be dictatorial (i.e., there is no dictator for $c$ ).

The last definitions introduce a new restriction on the type of choice functions which are generated by a social choice rule acting on agendas. First of all, we define the concept of a rational choice function. Then we define what is meant by a social choice rule having a rational explanation.

Definition 9. A choice function $c$ on a set $X$ is called rational if there exists a total preorder $\succsim$ on $X$ such that $c(v)=\{x \in v: x \succsim y$ holds for every $y \in v\}$.

A social choice rule is said to have a rational explanation if any choice function in its range is rational. 
There is a lot of other classical properties for social rules (e.g., anonymity). However, we stop at this stage: the properties defined above are already enough to obtain a key result about social choice models to fuse individual preferences.

Theorem 2 (Arrow's impossibility theorem (1950), see [1-3]). When there are at least three alternatives $(|X| \geq 3)$ as well as at least three individuals in the society $(|N| \geq 3)$, there is no social choice rule $c$ on a set $X$ satisfying the following list of properties: $c$ has universal domain, $c$ is Paretian, $c$ satisfies the condition of independence of irrelevant alternatives, $c$ has a rational explanation, $c$ satisfies non-dictatorship.

Arrow's theorem is a cornerstone in Social Choice. It broke the previous paradigm: most people was expecting some mathematical result that could help any decision-maker to find good social choice rules in any Social Choice model. The impossibility shown by the famous Arrovian theorem was overwhelming. Arrow's impossibility theorem also has many variants that involve slight changes in its statement and/or in the restrictions imposed on the rules. To fix ideas, we include here one variant due to Peter C. Fishburn, and issued in 1970 (see [23]).

Theorem 3. Let $X$ stand for a finite set whose cardinality is at least three. Let $n \geq 3 \in \mathbb{N}$. Let $\mathcal{A}$ stand for the set of n-profiles of preferences on $X$. Denote by $\mathcal{O}$ the family of the asymmetric parts of all the total preorders defined on $X$. Then there is no map $F: \mathcal{O}^{n} \longrightarrow \mathcal{O}$ satisfying the following properties:

(i) Unanimity: For every $x, y \in X$ and every profile $\mathcal{P}=\left(\succsim_{1}, \ldots, \succsim_{n}\right) \in \mathcal{A}$ such that $x \succ_{i} y$ holds for every $1 \leq i \leq n$, it holds that $x F(\mathcal{P}) y$,

(ii) Independence of irrelevant alternatives (Fishburn's version): For any $x, y \in X$ and $\mathcal{P}=\left(\succsim_{1}, \ldots, \succsim_{n}\right.$ )$; \mathcal{P}^{\prime}=\left(\succsim_{1}^{\prime}, \ldots, \succsim_{n}^{\prime}\right) \in \mathcal{A}$ if the restrictions to $\{x, y\}$ of $\succ_{i}$ and $\succ_{i}^{\prime}$ agree for all $1 \leq i \leq n$, then $x F(\mathcal{P}) y=x F\left(\mathcal{P}^{\prime}\right) y$.

(iii) Non-dictatorship: There is no $k \in\{1, \ldots n\}$ such that for every $x, y \in X$ and $\mathcal{P}=\left(\succsim_{1}, \ldots, \succsim_{n}\right) \in \mathcal{A}$ it holds that $x \succ_{k} y \Rightarrow x F(\mathcal{P}) y$.

Proof. See the main result in [23]. (For an alternative but similar setting and proof, see [24]).

\subsection{Fuzzy Sets}

Henceforward $U$ will denote a nonempty set, also known as the universe.

Definition 10. A fuzzy subset $X$ of $U$ is defined as a function $\mu_{X}: U \rightarrow[0,1]$. The function $\mu_{X}$ is called the membership function of $X$. In the particular case when $\mu_{X}$ is dichotomic and takes values in $\{0,1\}$, the corresponding subset defined by means of $\mu_{X}$ is a subset of $U$ in the classical crisp sense (the term 'crisp' is usually understood in these contexts as meaning non-fuzzy). For any $\alpha \in[0,1]$ we define the $\alpha$-cut of $X$ as the crisp subset of the universe $U$ given by $X_{\alpha}=\left\{t \in U: \mu_{X}(t) \geq \alpha\right\}$.

The support of $X$ is the crisp subset $\operatorname{Supp}(X)=\left\{t \in U: \mu_{X}(t) \neq 0\right\} \subseteq U$, whereas the kernel of $X$ is the crisp subset $\operatorname{Ker}(X)=\left\{t \in U: \mu_{X}(t)=1\right\} \subseteq U$. The fuzzy set $X$ is said to be normal if its kernel is nonempty.

Definition 11. A fuzzy binary relation defined on $U$ is a bivariate map $\mathcal{F}: U \times U \rightarrow[0,1]$, that is, $\mathcal{F}$ defines a fuzzy subset of the Cartesian product $U \times U$. Notice that we write directly $\mathcal{F}$ instead of $\mu_{\mathcal{F}}$, so that $\mathcal{F}$ directly acts as a membership function of a fuzzy subset of $U \times U$. In fact, the corresponding fuzzy subset is also denoted $\mathcal{F}$ if this does not lead to a misunderstanding.

Definition 12. Let $\mathcal{F}$ and $\mathcal{F}^{\prime}$ be two fuzzy binary relations on a universe $U$. We say that $\mathcal{F}$ is equivalent to $\mathcal{F}^{\prime}$, and we denote this by $\mathcal{F} \approx \mathcal{F}^{\prime}$ if the supports of $\mathcal{F}$ and $\mathcal{F}^{\prime}$ coincide and, in addition, there exists an order isomorphism $g: \mathcal{F}(U \times U) \longrightarrow \mathcal{F}^{\prime}(U \times U)$ such that for every $\alpha \in \mathcal{F}(U \times U)$ the $\alpha$-cut $\mathcal{F}_{\alpha}$ coincides with the $g(\alpha)$-cut $\mathcal{F}_{g(\alpha)}^{\prime}$. 
Proposition 1. Let $\mathcal{F}$ and $\mathcal{F}^{\prime}$ be two fuzzy binary relations on a universe $U$. Then $\mathcal{F} \approx \mathcal{F}^{\prime}$ holds if and only if $\operatorname{Supp}(\mathcal{F})=\operatorname{Supp}\left(\mathcal{F}^{\prime}\right)$ and, in addition, for all $\bar{z}=(a, b) ; \bar{z}^{\prime}=\left(a^{\prime}, b^{\prime}\right) \in U \times U$ we have that $\mathcal{F}(\bar{z})<\mathcal{F}\left(\bar{z}^{\prime}\right) \Leftrightarrow \mathcal{F}^{\prime}(\bar{z})<\mathcal{F}^{\prime}\left(\bar{z}^{\prime}\right)$.

Proof. First observe that the condition on the respective supports is common in both sides of the equivalence implication. Now suppose that $\mathcal{F} \approx \mathcal{F}^{\prime}$. Then there exists an order isomorphism $g: \mathcal{F}(U \times U) \longrightarrow \mathcal{F}^{\prime}(U \times U)$ accomplishing that $\mathcal{F}_{\alpha}=\mathcal{F}_{g(\alpha)}^{\prime}$ holds for every $\alpha \in \mathcal{F}(U \times U)$. If $\mathcal{F}(\bar{z})<\mathcal{F}\left(\bar{z}^{\prime}\right)$, then $\bar{z} \notin \mathcal{F}_{\mathcal{F}\left(\bar{z}^{\prime}\right)}=\mathcal{F}_{g\left(\mathcal{F}\left(\bar{z}^{\prime}\right)\right)^{\prime}}^{\prime}$ but $\bar{z}^{\prime} \in \mathcal{F}_{\mathcal{F}\left(\bar{z}^{\prime}\right)}=\mathcal{F}_{g\left(\mathcal{F}\left(\bar{z}^{\prime}\right)\right)}^{\prime}$, so $\mathcal{F}^{\prime}(\bar{z})<\mathcal{F}^{\prime}\left(\bar{z}^{\prime}\right)$. We can proceed equivalently with $g^{-1}$ to obtain $\mathcal{F}^{\prime}(\bar{z})<\mathcal{F}^{\prime}\left(\bar{z}^{\prime}\right) \Rightarrow \mathcal{F}(\bar{z})<\mathcal{F}\left(\bar{z}^{\prime}\right)$.

To prove the converse implication we define $g(t)$ (with $t \in \mathcal{F}(U \times U)$ ) as the unique value $s \in \mathcal{F}^{\prime}(U \times U)$ such that $\mathcal{F}_{t}=\mathcal{F}_{s}^{\prime}$. Such a value exists because there is a $\bar{z}_{0} \in U \times U$ with $\mathcal{F}\left(\bar{z}_{0}\right)=t$, so $\mathcal{F}_{t}=\left\{\bar{z} \in U \times U: \mathcal{F}\left(\bar{z}_{0}\right) \leq \mathcal{F}(\bar{z})\right\}=\left\{\bar{z} \in U \times U: \mathcal{F}^{\prime}\left(\bar{z}_{0}\right) \leq \mathcal{F}^{\prime}(\bar{z})\right\}=\mathcal{F}_{\mathcal{F}^{\prime}\left(\bar{z}_{0}\right)}^{\prime}$. Notice that, if there exists another value $s^{\prime}=\mathcal{F}^{\prime}\left(\bar{z}_{1}\right) \in \mathcal{F}^{\prime}(U \times U)$ with $\mathcal{F}_{t}=\mathcal{F}_{s^{\prime}}^{\prime}$, then, as $\bar{z}_{i} \in \mathcal{F}^{\prime}{ }_{\mathcal{F}^{\prime}\left(\bar{z}_{i}\right)}$ for $i=0,1$, from $\mathcal{F}^{\prime}{ }_{\mathcal{F}^{\prime}\left(\bar{z}_{0}\right)}=\mathcal{F}_{t}=\mathcal{F}^{\prime}{ }_{\mathcal{F}^{\prime}\left(\bar{z}_{1}\right)}$ we would obtain that $\bar{z}_{1} \in \mathcal{F}^{\prime}{ }_{s}$ and also $\bar{z}_{0} \in \mathcal{F}^{\prime}{ }_{s^{\prime}}$, so concluding that $s \leq \mathcal{F}^{\prime}\left(\bar{z}_{1}\right)=s^{\prime} \leq \mathcal{F}^{\prime}\left(\bar{z}_{0}\right)=s$. Moreover, if $t<t^{\prime}$ then $\mathcal{F}_{t^{\prime}} \subsetneq \mathcal{F}_{t}$, so that $\mathcal{F}^{\prime}{ }_{s^{\prime}} \subsetneq \mathcal{F}^{\prime}{ }_{s}$. Hence $s<s^{\prime}$ and $g$ is increasing, preserving the usual order $\leq$ of the real line. Bearing all this in mind we can define analogously an order-preserving map $h: \mathcal{F}^{\prime}(U \times U) \longrightarrow \mathcal{F}(U \times U)$ such that $g$ and $h$ are inverses one another.

\section{Decomposition of Fuzzy Binary Relations}

Now we are interested in generalizing to the fuzzy setting the notion of decomposition of a binary relation (see Definition 2 above).

To start with, we extend the notions of symmetry and asymmetry. The following definition has been adopted by several authors (see e.g., $[7,8,25,26])$.

Definition 13. A fuzzy binary relation $\mathcal{R}$ on a universe $U$ is said to be symmetric if $\mathcal{R}(x, y)=\mathcal{R}(y, x)$ holds true for every $x, y \in U$. $\mathcal{R}$ is called antisymmetric if for any $x, y \in U, \mathcal{R}(x, y)>0$ implies that $\mathcal{R}(y, x)=0$.

Now we need to extend to the fuzzy setting the concept of union of sets.

Definition 14. A fuzzy union -in the literature this is also called a semi-t-conorm or dual to a semi-copulais a binary operation $\cup_{f}:[0,1] \times[0,1] \rightarrow[0,1]$ that satisfies the following properties (\{here we will use the classical notation $\}$, so that $\cup_{f}(a, b)$ is usually denoted as $\left.a \cup_{f} b(a, b \in[0,1])\right)$ :

(i) Boundary conditions: For any $t \in[0,1]$, it holds that $0 \cup_{f} t=t \cup_{f} 0=t$.

(ii) Monotonicity: For all $a, b, c, d \in[0,1]$ with $a \leq c$ and $b \leq d$ it holds true that $a \cup_{f} b \leq c \cup_{f} d$.

Given two fuzzy binary relations $\mathcal{R}$ and $\mathcal{R}^{\prime}$ on the same universe $U$, its union as regards $\cup_{f}$ is the new binary relation $\mathcal{R} \cup_{f} \mathcal{R}^{\prime}$, defined as follows: $\mathcal{R} \cup_{f} \mathcal{R}^{\prime}(x, y)=\mathcal{R}(x, y) \cup_{f} \mathcal{R}^{\prime}(x, y)$, for every $x, y \in U$.

Remark 1. Given a fuzzy union $\cup_{f}$ we may observe that its restriction to $\{0,1\}$ is $0 \cup_{f} 0=0$, whereas $0 \cup_{f} 1=1 \cup_{f} 0=1 \cup_{f} 1=1$. This corresponds to the following important fact: In the crisp setting the membership function of a union $A \cup B$ of two sets satisfies that, for every $x \in A \cup B, \mu_{A \cup B}(x)=0$ if and only if $\mu_{A}(x)=\mu_{B}(x)=0$, whereas $\mu_{A \cup B}(x)=1$ otherwise.

Definition 15. Given a fuzzy union $\cup_{f}$, and a fuzzy binary relation $\mathcal{R}$ defined on a universe $U$, we say that $\mathcal{R}$ is decomposable with respect to $\cup_{f}$ if there exists a fuzzy asymmetric relation $\mathcal{P}$ and a fuzzy symmetric relation $\mathcal{I}$ such that $\mathcal{R}=\mathcal{P} \cup \mathcal{I}$. We denote it as $\mathcal{R}=\{\mathcal{P}, \mathcal{I}\}$. Here $\{\mathcal{P}, \mathcal{I}\}$ is the corresponding decomposition of $\mathcal{R}$.

At this stage we wonder if given a fuzzy union $\cup_{f}$ it is possible to decompose any fuzzy relation $\mathcal{R}$ on a universe $U$ as $\{\mathcal{P}, \mathcal{I}\}$, with $\mathcal{P}$ asymmetric and $\mathcal{I}$ symmetric. Assuming that this is possible, we may also ask ourselves if the decomposition is unique or not. 
The answers to both questions, namely the existence and uniqueness of decompositions, are negative. Check the Examples 1 and 2 below. Before showing those examples, we need to introduce the following proposition.

Proposition 2. Let $\cup_{f}$ be a fuzzy union. Let $\mathcal{R}$ be a fuzzy binary relation defined on a universe $U$. Assume that $\mathcal{R}$ admits a decomposition $\{\mathcal{P}, \mathcal{I}\}$, with $\mathcal{P}$ asymmetric and $\mathcal{I}$ symmetric. Then, for any $(x, y) \in U \times U$ it holds that $\mathcal{I}(x, y)=\min \{\mathcal{R}(x, y), \mathcal{R}(y, x)\}$.

Proof. Once a pair $x, y \in X$ has been fixed, without loss of generality we can suppose that $\mathcal{R}(x, y) \geq$ $\mathcal{R}(y, x)$, so $\mathcal{R}(y, x)=\min \{\mathcal{R}(x, y), \mathcal{R}(y, x)\}$. By decomposability of $\mathcal{R}$ into $\mathcal{P}$ and $\mathcal{I}$ we have that $\mathcal{P}(x, y) \cup_{f} \mathcal{I}(x, y)=\mathcal{R}(x, y) \geq \mathcal{R}(y, x)=\mathcal{P}(x, y) \cup_{f} \mathcal{I}(x, y)$. By asymmetry of $\mathcal{P}$ it follows that $\mathcal{P}(x, y)=0$ or $\mathcal{P}(y, x)=0$. Thus, if $\mathcal{P}(y, x)=0$ then we have that $\mathcal{I}(x, y)=\mathcal{R}(y, x)$, whereas if $\mathcal{P}(x, y)=0$, then $\mathcal{I}(x, y)=\mathcal{I}(x, y) \cup_{f} \mathcal{P}(x, y)=\mathcal{R}(x, y) \geq \mathcal{R}(y, x)$. By monotonicity of $\cup_{f}$, we conclude that $\mathcal{I}(x, y)=\mathcal{I}(x, y) \cup_{f} \mathcal{P}(x, y) \leq \mathcal{I}(x, y) \cup_{f} \mathcal{P}(y, x)=\mathcal{R}(y, x)$. Therefore $\mathcal{I}(x, y)=$ $\mathcal{R}(y, x)$.

Proposition 2 is crucial because it establishes that if there exists a decomposition, the symmetric part will always be the same, independently of the fuzzy union $\cup_{f}$ considered.

Let us see now two examples illustrating that decompositions do not always exist, and, in case of existence, they are not necessarily unique.

Example 1. Consider the drastic union $\cup_{D}$ defined for every $a, b \in[0,1]$ as: $a \cup_{D} b=a$ if $b=0 ; a \cup_{D} b=b$ if $a=0 ; a \cup_{D} b=1$ otherwise.

For such union $\cup_{D}$ not every fuzzy binary relation is decomposable. To see this, consider a relation $\mathcal{R}$ defined on a universe $U$ and such that $\mathcal{R}(x, y)=0.7$ and $\mathcal{R}(y, x)=0.3$ for any $x \neq y \in X$. If there exist a decomposition $\{\mathcal{P}, \mathcal{I}\}$ of $\mathcal{R}$, then by Proposition 2 it holds that $\mathcal{I}(x, y)=0.3$ : However there is no value $t \in[0,1]$ such that $0.7=0.3 \cup_{D}$ t. So $\mathcal{R}$ can not be decomposed.

Example 2. Consider the $₫ u k a s i e w i c z$ union $\cup_{\ell}$ given by $a \cup_{\ell} b=\min \{1, a+b\}$. Given a fuzzy relation $\mathcal{R}$ on a universe $U$, and such that for some pair $(x, y) \in U \times U$ it holds that $\mathcal{R}(x, y)=1$ and $\mathcal{R}(y, x)=0.9$. Notice that $\mathcal{R}$ can be decomposed as $\{\mathcal{P}, \mathcal{I}\}$ with: $\mathcal{I}(a, b)=\min \{\mathcal{R}(a, b), \mathcal{R}(b, a)\}$, and $\mathcal{P}(a, b)=\mathcal{R}(a, b)-$ $\mathcal{R}(b, a)$ if if $\mathcal{R}(a, b)>\mathcal{R}(b, a) ; \mathcal{P}(a, b)=0$ otherwise. $(a, b \in U)$.

However, we can find another decomposition $\left\{\mathcal{P}^{\prime}, \mathcal{I}\right\}$, with $\mathcal{I}$ given as before, and $\mathcal{P}^{\prime}$ defined by: $\mathcal{P}^{\prime}(a, b)=$ 1 if $1=\mathcal{R}(a, b)>\mathcal{R}(b, a) ; \mathcal{P}^{\prime}(a, b)=\mathcal{R}(a, b)-\mathcal{R}(b, a)$ if $1>\mathcal{R}(a, b)>\mathcal{R}(b, a)$, and $\mathcal{P}^{\prime}(a, b)=0$ otherwise. $(a, b \in U)$.

It is plain that these decompositions of $\mathcal{R}$ are different, because $\mathcal{P}(x, y)=0.1$ whilst $\mathcal{P}^{\prime}(x, y)=1$.

Now we search for necessary and sufficient conditions that guarantee both existence and uniqueness of decompositions of fuzzy binary relations.

Proposition 3. Let $\cup_{f}$ be a fuzzy union and $U$ a universe. Every fuzzy relation $\mathcal{R}$ on $U$ is decomposable if and only if $\cup_{f}$ is continuous on the second coordinate (with respect to the Euclidean topology on the unit interval $[0,1])$. If $\cup_{f}$ is strictly increasing on the second coordinate then every decomposable fuzzy relation has a unique decomposition.

Proof. First of all we will introduce an auxiliary new operation, denoted $\searrow$ and defined as follows: given $a, b \in[0,1], a \searrow b$ is defined as $\inf \left\{t \in[0,1]: a \cup_{f} t \geq b\right\}$. In addition, for each $a \in[0,1]$ we define the function $f_{a}:[0,1] \rightarrow[0,1]$, as follows $f_{a}(t)=a \cup t(t \in[0,1])$.

If $\cup_{f}$ is continuous in each coordinate, then $f_{a}$ is continuous for every $a$ in the unit interval. Given a fuzzy relation $\mathcal{R}$ on the universe $U$, by Proposition 2 we may already define the symmetric part of any possible decomposition of $\mathcal{R}$ as the fuzzy binary relation $\mathcal{I}$ given by $\mathcal{I}(x, y)=\min \{\mathcal{R}(x, y), \mathcal{R}(y, x)\}$. 
In addition we consider the fuzzy binary relation $\mathcal{P}$ given by $\mathcal{P}(x, y)=\mathcal{I}(x, y) \searrow \mathcal{R}(x, y)$, for every $(x, y) \in U \times U$. We claim that $\{\mathcal{P}, \mathcal{I}\}$ is a decomposition of $\mathcal{R}$. To see this, it is enough to check that $\mathcal{R}=\mathcal{P} \cup_{f} \mathcal{I}$. Thus, given $x, y \in U$, if $\mathcal{R}(x, y) \leq \mathcal{R}(y, x)$, then, by definition, we have that $\mathcal{I}(x, y)=$ $\mathcal{R}(x, y)$ and $\mathcal{P}(x, y)=\mathcal{I}(x, y) \searrow \mathcal{R}(x, y)=0$, so $\mathcal{R}(x, y)=\mathcal{I}(x, y) \cup_{f} \mathcal{P}(x, y)$. If $\mathcal{R}(x, y)>\mathcal{R}(y, x)$, then it holds that $\mathcal{I}(x, y)=\mathcal{R}(y, x)$ and $\mathcal{P}(x, y)=\inf \left\{t \in[0,1]: f_{\mathcal{R}(y, x)}(t) \geq \mathcal{R}(x, y)\right\}$. Notice that $f_{\mathcal{R}(y, x)}(0)=\mathcal{R}(y, x)<\mathcal{R}(x, y)$ and $f_{\mathcal{R}(y, x)}(1)=1$. By continuity of $f_{\mathcal{R}(y, x)}$ there exists an $\alpha \in[0,1]$ with $f_{\mathcal{R}(y, x)}(\alpha)=\mathcal{R}(x, y)$. Since $\mathcal{R}(x, y)$ is attained by $f_{\mathcal{R}(y, x)}$ and $f_{\mathcal{R}(y, x)}$ is continuous, we conclude that $f_{\mathcal{R}(y, x)}(\mathcal{P}(x, y))=\mathcal{R}(x, y)$.

Suppose now that every binary fuzzy relation on the universe $U$ is decomposable. We will prove first that for every $a \in[0,1]$, it holds that $f_{a}([0,1])=[a, 1]$. Next we will prove the continuity of $f_{a}$. To see all this, given any $s \in[a, 1]$ we consider a fuzzy relation $\mathcal{R}$ with $\mathcal{R}(x, y)=s$ and $\mathcal{R}(y, x)=a$ for some $x, y \in U$. By decomposability there exists a pair $(\mathcal{P}, \mathcal{I})$ with $\mathcal{R}=\mathcal{P} \cup_{f} \mathcal{I}$. By Proposition 2 it follows that $\mathcal{I}(x, y)=a$. Hence $s=\mathcal{R}(x, y)=f_{a}(\mathcal{P}(x, y))$.

To conclude the argument, assume that $f_{a}$ is discontinuous. Then, in $[0,1]$ there exist a sequence $\left(t_{n}\right)$ converging to $t \in[0,1]$ and such that $\left(f_{a}\left(t_{n}\right)\right)$ does not converge to $f_{a}(t)$. We may assume without loss of generality that such sequence is increasing (or decreasing) subsequence of $\left(t_{n}\right)$ bounded above (or below) by $t$. We will assume here that it is indeed increasing (an similar argument would be used by the decreasing case). By monotonicity $\left(f_{a}\left(t_{n}\right)\right)$ is increasing and bounded above by $f_{a}(t)$, so it converges to some value $\lambda<f_{a}(t)$. Since $f_{a}([0,1])=[a, 1]$, there exists a value $w \in[0,1]$ with $\lambda<f_{a}(w)<f_{a}(t)$. By monotonicity $t_{n}<w<t$, but $t_{n} \rightarrow t$, so we arrive at a contradiction. Therefore $f_{a}$ is a continuous function.

Suppose, finally, that $\cup_{f}$ is strictly increasing on the second coordinate. Let $\mathcal{R}$ be a fuzzy relation on the universe $U$. Assume that $\mathcal{R}$ admits two different decompositions, namely $\{\mathcal{P}, \mathcal{I}\}$ and $\left\{\mathcal{P}^{\prime}, \mathcal{I}^{\prime}\right\}$. By Proposition 2 we have that $\mathcal{I}=\mathcal{I}^{\prime}$, so the asymmetric parts $\mathcal{P}$ and $\mathcal{P}^{\prime}$ should be different. Hence there exist $x, y \in U$ such that $\mathcal{P}(x, y) \neq \mathcal{P}^{\prime}(x, y)$. We may assume, without loss of generality, that $\mathcal{P}(x, y)<\mathcal{P}^{\prime}(x, y)$. Since $\cup_{f}$ is strictly increasing on the second coordinate we arrive at the fact $\mathcal{R}(x, y)=\mathcal{I}(x, y) \cup_{f} \mathcal{P}(x, y)<\mathcal{I}^{\prime}(x, y) \cup \mathcal{P}^{\prime}(x, y)=\mathcal{R}(x, y)$. This is a clear contradiction, so that, a fortiori, the decomposition is unique.

To conclude this section we introduce some properties of the (unique) decompositions that come from a fuzzy union $\cup_{f}$ that is continuous and strictly increasing on the second coordinate.

Proposition 4. Let $\mathcal{R}$ be a fuzzy binary relation on the universe $U$. Let $\cup_{f}$ be a continuous fuzzy union that is strictly increasing on the second coordinate. Let $\{\mathcal{P}, \mathcal{I}\}$ stand for the unique decomposition of $\mathcal{R}$ as regards $\cup_{f}$. Then following properties hold true:

(i) $\mathcal{P}(x, y) \leq \mathcal{R}(x, y)$ for all $x, y \in U$,

(ii) $\mathcal{P}(x, y)=0 \Rightarrow \mathcal{R}(x, y)=\mathcal{I}(x, y)$, for any $x, y \in U$,

(iii) $[\mathcal{I}(x, y) \leq \mathcal{I}(z, w)] \wedge[\mathcal{P}(x, y) \leq \mathcal{P}(z, w)] \Rightarrow[\mathcal{R}(x, y) \leq \mathcal{R}(z, w)]$ holds true for every $x, y, z, w \in U$, (iv) $\mathcal{P}(x, y)>0 \Leftrightarrow \mathcal{R}(x, y)>\mathcal{R}(y, x)$ for every $x, y \in U$.

Proof. To prove (i) notice that $\mathcal{R}(x, y)=\mathcal{I}(x, y) \cup_{f} \mathcal{P}(x, y) \geq 0 \cup_{f} \mathcal{P}(x, y)=\mathcal{R}(x, y)$. To prove (ii) notice that if $\mathcal{P}(x, y)=0$, then $\mathcal{I}(x, y)=\mathcal{I}(x, y) \cup_{f} \mathcal{P}(x, y)=\mathcal{I}(x, y) \cup 0=\mathcal{I}(x, y)$. Property (iii) is a direct consequence of the monotonicity of $\cup_{f}$. Finally, to prove (iv) we may observe that if $\mathcal{P}(x, y)>0$, then $\mathcal{P}(y, x)=0$ because $\mathcal{P}$ is asymmetric. Hence $\mathcal{R}(x, y)=\mathcal{I}(x, y) \cup_{f} \mathcal{P}(x, y)>$ $\mathcal{I}(y, x) \cup_{f} 0=\mathcal{R}(y, x)$. Conversely, if $\mathcal{R}(x, y)>\mathcal{R}(y, x)$, by property (iii) it follows now that $[\mathcal{I}(x, y)>$ $\mathcal{I}(y, x)] \vee[\mathcal{P}(x, y)>\mathcal{P}(y, x)]$ holds true. However, since $I$ is symmetric, so that $\mathcal{I}(x, y)=\mathcal{I}(y, x)$, we get $\mathcal{P}(x, y)>\mathcal{P}(y, x) \geq 0$.

Given a fuzzy union $\cup_{f}$, it may happen that it is discontinuous, or it fails to be strictly increasing with respect to the second coordinate. When this happens, a fuzzy relation $\mathcal{R}$ on a universe $U$ may or may not give rise to decompositions. When they exist, it may also happen that there is more than one. This suggests the following definition. 
Definition 16. Let $\mathcal{R}$ be a fuzzy binary relation on the universe $U$. Let $\cup_{f}$ be a fuzzy union. Assume that $\mathcal{R}$ admits a decomposition $\{\mathcal{P}, \mathcal{I}\}$ as regards $\cup_{f}$, so that $\mathcal{P}($ respectively, $\mathcal{I})$ is asymmetric (respectively, symmetric). Then the decomposition $\{\mathcal{P}, \mathcal{I}\}$ is said to be admissible if it satisfies the properties (i) to (iv) that appear in the statement of Proposition 4.

\section{Fuzzy Preferences}

As aforesaid, in the classical crisp models in social choice a preference is understood as a total preorder. Bearing in mind that a (total) preorder $\succsim$ on a set $X$ can be decomposed through the pair $\{\succ, \sim\}$, we may think of fuzzy preferences as fuzzy binary relations $\mathcal{R}$ on a universe $U$, such that $\mathcal{R}$ can be decomposed someway into other fuzzy binary relations $\mathcal{P}$, and $\mathcal{I}$ so that $\mathcal{R}$ (respectively: $\mathcal{P}, \mathcal{I}$ ) plays the role of a weak preference (respectively: of a strict preference, of an indifference), as $(\succsim, \succ, \sim)$ in the classical approach. This question has special relevance. Several authors have already explored this topic with particular decompositions (see, e.g., $[7,8]$ ). Other authors have worked under the axiomatic existence of decompositions (see [19]).

In addition, a look to Proposition 3 shows that we have characterized there when every fuzzy binary relation is decomposable into an asymmetric and symmetric relations. In practice, perhaps we are not interested in decomposing any relation, but, instead, a particular one with some suitable features. Hence, the problem of how to decompose a given fuzzy binary relation is, in general, different from that of decomposing every one.

Even when a given fuzzy binary relation $\mathcal{R}$ admits a decomposition into an asymmetric $\mathcal{P}$ and a symmetric one $\mathcal{I}$, we may be interested in certain additional properties that $\mathcal{P}$ or $\mathcal{I}$ may or may not satisfy.

Example 3. Consider the maximum as a fuzzy union. It is a continuous function, so by Proposition 3 each fuzzy binary relation on a universe $U$ admits decompositions. However, they are not unique because the maximum is not strictly increasing on the second coordinate. Consider first the decomposition of a fuzzy relation $\mathcal{R}$ obtained by the technique introduced the proof of Proposition 3. Thus, we have that $\mathcal{I}(x, y)=\min \{\mathcal{R}(x, y), \mathcal{R}(y, x)\}$, and $\mathcal{P}(x, y)=\mathcal{R}(x, y)$ if $\mathcal{R}(x, y)>\mathcal{R}(y, x), \mathcal{R}(x, y)=0$ otherwise, $(x, y \in X)$. This is not the only possible decomposition in this case. For example, if we fix an element $a$ in the universe $U$ we can consider other decomposition, $\left\{\mathcal{P}^{\prime}, \mathcal{I}\right\}$ defined as follows: $\mathcal{P}^{\prime}(x, y)=\mathcal{R}(x, y)$ if $\mathcal{R}(x, y)>\mathcal{R}(y, x), \quad \mathcal{R}(x, y)=\frac{\mathcal{R}(a, y)}{2}$ if $x=a, y \neq a$ and $\mathcal{R}(x, y)=\mathcal{R}(y, x), \quad \mathcal{R}(x, y)=0$ otherwise, $(x, y \in X)$. Fortunately, we can add some reasonable additional property to provoke that there is only one decomposition under the maximum fuzzy union that satisfies those added requirements. For instance, in several contexts it is reasonable to request that if $\mathcal{R}(x, y)>\mathcal{R}(y, x)$ then $(\mathcal{P}(x, y)>0)$, and conversely, so that a suitable restriction could be to request that for any fuzzy binary relation $\mathcal{R}$ on $U$ and $x, y \in U$ it holds that $\mathcal{R}(x, y)>\mathcal{R}(y, x) \Leftrightarrow \mathcal{P}(x, y)>0$. (We may have something of this kind in mind because $\mathcal{R}(x, y)$ would represent how much $x$ is preferred over $y)$. Here the first decomposition suggested in this example accomplishes this restriction, but the second one does not. As a matter of fact it is straightforward to prove that the first decomposition is the unique one that satisfies the aforementioned requirement.

Let us give an account of all the generalizations to be made when passing from crisp preferences to fuzzy preferences. If we understand a crisp preference as a total preorder $\succsim$ on a set $X$, but immediately take into account that $\succsim=\succ \cup \sim$, in order to work with the triplet $(\succsim, \succ, \sim)$ when necessary, we may realize that in the fuzzy setting we should start with a binary relation $\mathcal{R}$ that accomplishes some properties that could remind us a total preorder, namely transitivity and completeness (that in the crisp setting implies reflexivity). However, in the fuzzy setting, many possible non-equivalent extensions of these concepts (see e.g., [27]) are at our disposal, so that we should choose a suitable one. The same happens with unions. As afore seen in Definition 14, many possible fuzzy unions are available. Again we should select one ad hoc. Finally, the concepts of asymmetry and symmetry have also been extended to the fuzzy setting in Definition 13. Once more, such a definition is not the only 
possible. Other non-equivalent definitions of, say, asymmetry are still possible. (For instance, we could think of an asymmetric fuzzy binary relation $\mathcal{R}$ on a universe $U$ as one that satisfies $\mathcal{R}(x, x)=0$ for every $x \in U$, and $\mathcal{R}(x, y)+\mathcal{R}(y, x)=1$ for every $x \neq y \in U)$.

Bearing all this in mind, we introduce the following definitions.

Definition 17. Let $\mathcal{R}$ be a fuzzy binary relation on a universe $U$. Let $\cup_{f}$ be a fuzzy union. The relation $\mathcal{R}$ is said to be:

(i) reflexive if for all $x \in U$ it holds that $\mathcal{R}(x, x)=1$,

(ii) transitive if for every $x, y, z \in U$ it holds that $[\mathcal{R}(x, y) \geq \mathcal{R}(y, x)] \wedge[\mathcal{R}(y, z) \geq \mathcal{R}(z, y)] \Rightarrow$ $[\mathcal{R}(x, z) \geq \mathcal{R}(z, x)]$

(iii) connected with respect to $\cup_{f}$ if for every pair $x, y \in U$ it holds that $\mathcal{R}(x, y) \cup_{f} \mathcal{R}(y, x)=1$,

(iv) complete if for every $x, y \in U$ it holds that $\max \{\mathcal{R}(x, y), \mathcal{R}(y, x)\}=1$,

(v) connected if $\mathcal{R}(x, y)+\mathcal{R}(y, x) \geq 1$ holds true for all $x, y \in U$.

Remark 2. The completeness (iv) and the connectedness (v) are already present in a vast literature (see $[7,8,19])$. Both are particular cases of $\cup_{f}$-connectedness for particular unions. For instance, given any union $\cup_{f}$ without divisors of 1 we may notice that to be $\cup_{f}$-connected is equivalent to completeness. $(a \in(0,1)$ is a divisor of 1 as regards a fuzzy union $\cup_{f}$ if there exist $a b \in(0,1)$ such that $\left.a \cup_{f} b=1\right)$. With respect to the Eukasiewicz

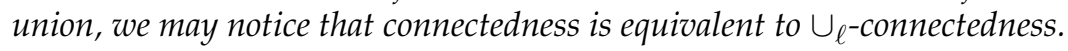

Furthermore, the fact of existence of different non-equivalent kinds of transitivity definitions and connectedness as well as completeness (see also [27-30]), tells us that the consideration in the fuzzy context of some kind of fuzzy total preorder is not unique. (Other non-equivalent definitions of transitivity have been introduced in this literature, see e.g., $[27,28,31,32])$. We should choose a suitable type.

Definition 18. Let $U$ be a universe and $\cup_{f}$ a fuzzy union. A fuzzy preference on $U$ relative to $\cup_{f}$ is a triplet $(\mathcal{R}, \mathcal{P}, \mathcal{I})$ of fuzzy binary relations, satisfying the following properties:

(i) $\mathcal{R}$ is reflexive, transitive and $\cup_{f}$-connected,

(ii) $\mathcal{P}$ is asymmetric, $\mathcal{I}$ is symmetric and $\mathcal{R}$ decomposes as $\{\mathcal{P}, \mathcal{I}\}$ with respect to $\cup_{f}$,

(iii) $\quad\{\mathcal{P}, \mathcal{I}\}$ is an admissible decomposition of $\mathcal{R}$.

The set of all fuzzy preferences on a universe $U$ will be denoted by $\mathcal{P}_{U}$.

Remark 3. Notice that Definition 18 extends to the fuzzy setting the concept of a (crisp) total preorder for $\mathcal{R}$ on the universe $U$. In fact, If $\mathcal{R}$ is a relation that only takes values in $\{0,1\}$, from reflexivity we see that $\mathcal{R}(x, x)=1$ for every $x \in U$. By $\cup_{f}$-connectedness, given $x, y \in U$ we have that $\mathcal{R}(x, y) \cup_{f} \mathcal{R}(y, x)=1$, which, by the properties of a fuzzy union and the fact of $\mathcal{R}$ taking values in $\{0,1\}$, implies that $\mathcal{R}(x, y)=1$ or $\mathcal{R}(y, x)=1$, so $\mathcal{R}$ is a complete (total) binary relation. By transitivity, given $x, y, z \in U$ from $\mathcal{R}(x, y)=1=\mathcal{R}(y, z)$ it finally follows that $\mathcal{R}(x, z) \geq \mathcal{R}(z, x)$. However, since $\mathcal{R}$ is already complete, this implies $\mathcal{R}(x, z)=1$. So $\mathcal{R}$ is also transitive. Hence it is a total preorder on $U$.

Conversely, if $\succsim$ stands for a total preorder defined on a universe $U$, it is clear, by Theorem 1 , that the triplet $(\succsim, \sim, \succ)$ actually satisfies Definition 18, so that it constitutes a particular case of a fuzzy preference.

\section{Arrow-Like Aggregation of Fuzzy Preferences}

In this section we extend to the fuzzy setting the concept of an Arrovian model. Therefore, we need to find suitable generalizations of the concepts arising in the classical (crisp) model to the new approach that involves fuzzy preferences. Then, with the help of a new technique based of the new concept of a pseudo-fuzzy preference, that is actually a bunch of five associated crisp binary relations, we will finally get an impossibility result à la Arrow, valid for fuzzy preferences.

As in the crisp case, we begin with the introduction of some necessary definition. 
Definition 19. Let $\mathcal{A} \subseteq \mathcal{P}_{U}$ be a nonempty set of fuzzy preferences defined on a universe $U$. Let $n \geq 3$ an integer number. A n-aggregation rule for fuzzy preferences is a function $f: \mathcal{A}^{n} \rightarrow \mathcal{A}$. Any element $\left(\Lambda_{1}, \ldots, \Lambda_{n}\right) \in \mathcal{A}^{n}$ is called a fuzzy profile, and it will be denoted by $\left(\Lambda_{i}\right)$ for short. Notice that for every $1 \leq i \leq n$, the fuzzy preference $\Lambda_{i}$ is a triplet of fuzzy binary relations $\left(\mathcal{R}_{i}, \mathcal{P}_{i}, \mathcal{I}_{i}\right)$ accomplishing the conditions introduced in Definition 18 when the concept of a fuzzy preference was launched. Similarly, $f\left(\left(\Lambda_{i}\right)\right)$ is also a triplet, that we will denote $\left(\mathcal{R}_{f}\left(\left(\Lambda_{i}\right)\right), \mathcal{P}_{f}\left(\left(\Lambda_{i}\right)\right), \mathcal{I}_{f}\left(\left(\Lambda_{i}\right)\right)\right)$. Besides, the set $\{1,2, \ldots, n\}$ will be denoted by $N$, and it is said to be the society, whose elements are called individuals or agents. The elements of the universe $U$ are called alternatives.

\subsection{The Fuzzy Arrovian Model}

Unlike the crisp Arrovian model considered in Section 2, in our fuzzy Arrovian model we will not deal with agendas and choice functions. Instead, we will directly deal with rules that aggregate individual fuzzy preferences, as introduced in Definition 18, into a new social one. We will ask the rules to accomplish some restrictions that look like some of the crisp Arrovian model, namely independence of irrelevant alternatives, a Paretian property and non-dictatorship. Concerning something that could remind us of the (crisp) condition of universal domain, we will assume that the $n$-rules that merge fuzzy preferences are defined on the whole $\mathcal{P}_{U}^{n}$. Finally, no condition similar to rational explanation is imposed a priori in the fuzzy approach.

Definition 20. Let $\mathcal{A} \subseteq \mathcal{P}_{U}$ stand for a nonempty set of fuzzy preferences defined on a universe $U$. A n-aggregation rule $f: \mathcal{A}^{n} \rightarrow \mathcal{A}$ is said to satisfy the property of:

(i) Independence of irrelevant alternatives if for any two profiles $\left(\Lambda_{i}\right)$ and $\left(\Lambda_{i}^{\prime}\right)$ that belong to $\mathcal{A}^{n}$ and $x, y \in U$ we have that if $\Lambda_{i\rceil\{x, y\}} \approx \Lambda_{i\rceil\{x, y\}}^{\prime}$ for any $i \in N$, then $f\left(\left(\Lambda_{i}\right)\right)_{\rceil\{x, y\}} \approx f\left(\left(\Lambda_{i}^{\prime}\right)\right)_{\rceil\{x, y\}}$. (Here $\Lambda_{i\rceil\{x, y\}}$ denotes the restriction of $\Lambda_{i}$ to the subset $\{x, y\}$ of alternatives. Given two profiles $\left(\Lambda_{i}\right)=\left(\mathcal{R}_{i}, \mathcal{P}_{i}, \mathcal{I}_{i}\right)$ and $\left(\Lambda_{i}^{\prime}\right)=\left(\mathcal{R}_{i}^{\prime}, \mathcal{P}_{i}^{\prime}, \mathcal{I}_{i}^{\prime}\right)$, the notation $\Lambda_{i} \approx \Lambda_{i}^{\prime}$ means that $\mathcal{R}_{i} \approx \mathcal{R}_{i}^{\prime}$ as well as $\mathcal{P}_{i} \approx \mathcal{P}_{i}^{\prime}$ and $\mathcal{I}_{i} \approx \mathcal{I}_{i}^{\prime}$, in the sense of Definition 12$)$.

(ii) Pareto if for every profile $\left(\Lambda_{i}\right) \in \mathcal{A}^{n}$ and any $x, y \in U$ it holds that if $\mathcal{P}_{i}(x, y)>0$ for any $i \in N$, then $\mathcal{P}_{f}\left(\left(\Lambda_{i}\right)\right)(x, y)>0$.

(iii) Dictatorship if there exists $k \in N_{n}$, called dictator, such that for every $\left(\Lambda_{i}\right)$ and $x, y \in U$ we have that if $\mathcal{P}_{k}(x, y)>0$ then $\mathcal{P}_{f}\left(\left(\Lambda_{i}\right)\right)(x, y)>0$.

\subsection{Pseudo-Fuzzy Preferences}

In order to tackle fuzzy preferences we will introduce a new technique: a fuzzy preference will be controlled by five closely associated crisp total preorders. The bunch of those five crisp relations that interpret a given fuzzy preference will be called a pseudo-fuzzy preference, see Definition 22 below. (We use the prefix "pseudo" because they are actually crisp (non-fuzzy)). To motivate that definition, we introduce a result related to equivalences of fuzzy preferences.

Proposition 5. Let $\Lambda=(\mathcal{R}, \mathcal{P}, \mathcal{I})$ and $\Lambda^{\prime}=\left(\mathcal{R}^{\prime}, \mathcal{P}^{\prime}, \mathcal{I}^{\prime}\right)$ be two fuzzy preferences on a universe $U$. Then for every $x, y \in U$ it holds that:

$$
\begin{gathered}
\Lambda_{\rceil\{x, y\}} \approx \Lambda_{\uparrow\{x, y\}}^{\prime} \Leftrightarrow\left\{\left[\mathcal{R}(a, b)>\mathcal{R}(b, a) \Leftrightarrow \mathcal{R}^{\prime}(a, b)>\mathcal{R}^{\prime}(b, a)\right]\right\} \wedge \\
\left.\wedge\left[\mathcal{R}(a, b)=0 \Leftrightarrow \mathcal{R}^{\prime}(a, b)=0\right] \wedge\left[\mathcal{R}(a, b)=1 \Leftrightarrow \mathcal{R}^{\prime}(a, b)=1\right](a, b \in\{x, y\})\right\} .
\end{gathered}
$$

Proof. First suppose that $\Lambda_{\rceil\{x, y\}} \approx \Lambda_{\rceil\{x, y\}}^{\prime}$. By Proposition 1 the first and second conditions follow immediately. Moreover, $\mathcal{R}(a, b)=1$, then $\mathcal{R}(a, b) \geq \mathcal{R}(x, x)$ and, again by Proposition 1 , it follows that $\mathcal{R}^{\prime}(a, b) \geq \mathcal{R}^{\prime}(x, x)=1$. Finally, the fact that $\mathcal{R}^{\prime}(a, b)=1 \Rightarrow \mathcal{R}(a, b)$ is proved in an analogous way. For the converse, notice that the second condition guarantees the equality of the corresponding supports, whereas the first one carries the equivalence $\mathcal{R}(\bar{z})<\mathcal{R}\left(\bar{z}^{\prime}\right) \Leftrightarrow \mathcal{R}^{\prime}(\bar{z})<\mathcal{R}^{\prime}\left(\bar{z}^{\prime}\right)$ for any $\bar{z}, \bar{z}^{\prime} \in$ 
$\{(x, y),(y, x)\}$. Finally, when $\bar{z}^{\prime} \in\{(x, x),(y, y)\}$ the corresponding equivalence is granted by the third condition, while if $\bar{z} \in\{(x, x),(y, y)\}$ it never happens that $\mathcal{R}(\bar{z})<\mathcal{R}\left(\bar{z}^{\prime}\right)$ nor $\mathcal{R}^{\prime}(\bar{z})<\mathcal{R}^{\prime}\left(\bar{z}^{\prime}\right)$.

Remark 4. In the spirit of Proposition 5, for each pair $(x, y) \in U \times U$ we can consider an equivalence relation, namely $\approx_{\{x, y\}}$ defined over the set $\mathcal{P}_{U}$ of fuzzy preferences on the universe $U$. Thus, given two fuzzy preferences $\Lambda, \Lambda^{\prime} \in \mathcal{P}_{U}$, we have that $\Lambda \approx_{\{x, y\}} \Lambda^{\prime} \Leftrightarrow \Lambda_{7\{x, y\}} \approx \Lambda_{\{\{x, y\}}^{\prime}$. Below we can see that the partition generated on the set of preferences $\mathcal{P}_{U}$ by $\approx_{\{x, y\}}$ consists of the following eight subsets:

$$
\begin{gathered}
\left\{(\mathcal{R}, \mathcal{P}, \mathcal{I}) \in \mathcal{P}_{U}: 1=\mathcal{R}(x, y)>\mathcal{R}(y, x)=0\right\}, \\
\left\{(\mathcal{R}, \mathcal{P}, \mathcal{I}) \in \mathcal{P}_{U}: 1=\mathcal{R}(x, y)>\mathcal{R}(y, x)>0\right\}, \\
\left\{(\mathcal{R}, \mathcal{P}, \mathcal{I}) \in \mathcal{P}_{U}: 1>\mathcal{R}(x, y)>\mathcal{R}(y, x)>0\right\}, \\
\left\{(\mathcal{R}, \mathcal{P}, \mathcal{I}) \in \mathcal{P}_{U}: 1=\mathcal{R}(x, y)=\mathcal{R}(y, x)\right\}, \\
\left\{(\mathcal{R}, \mathcal{P}, \mathcal{I}) \in \mathcal{P}_{U}: 1>\mathcal{R}(x, y)=\mathcal{R}(y, x)\right\}, \\
\left\{(\mathcal{R}, \mathcal{P}, \mathcal{I}) \in \mathcal{P}_{U}: 0<\mathcal{R}(x, y)<\mathcal{R}(y, x)<1\right\}, \\
\left\{(\mathcal{R}, \mathcal{P}, \mathcal{I}) \in \mathcal{P}_{U}: 1<\mathcal{R}(x, y)<\mathcal{R}(y, x)=1\right\}, \\
\left\{(\mathcal{R}, \mathcal{P}, \mathcal{I}) \in \mathcal{P}_{U}: 0=\mathcal{R}(x, y)<\mathcal{R}(y, x)=1\right\}
\end{gathered}
$$

Notice that depending on the pair $(x, y)$, some components could be empty. For instance, since the fuzzy preferences in $\mathcal{P}_{U}$ are reflexive, we have that for every $x \in U$ the partition induced by $(x, x)$ only consists of the whole set $\left\{\mathcal{P}_{U}\right\}$.

Bearing in mind the above partition, we introduce next notation and definition. Thus, let us suppose that an individual (or agent in the society) has defined the fuzzy preference $\Lambda=(\mathcal{R}, \mathcal{P}, \mathcal{I}) \in \mathcal{P}_{U}$. Given two alternatives $x, y$ in the universe $U$,

(i) if $1=\mathcal{R}(x, y)$ whereas $\mathcal{R}(y, x)=0$, we denote it by $x \succ_{\Lambda}^{+} y$,

(ii) if $1=\mathcal{R}(x, y)>\mathcal{R}(y, x)>0$, we denote it by $x \succ_{\Lambda}^{0} y$,

(iii) if $1>\mathcal{R}(x, y)>\mathcal{R}(y, x)>0$, we denote it by $x \succ_{\Lambda}^{-} y$,

(iv) if $1=\mathcal{R}(x, y)=\mathcal{R}(y, x)$, we denote it by $x \sim_{\Lambda}^{+} y$,

(v) if $1>\mathcal{R}(x, y)=\mathcal{R}(y, x)$, we denote it by $x \sim_{\Lambda}^{0} y$.

Hence we have got from $\Lambda$ five crisp binary relations on the universe $U$, namely $\left\{\succ_{\Lambda^{\prime}}^{+} \succ_{\Lambda^{\prime}}^{0} \succ_{\Lambda}^{-}\right.$ ,$\left.\sim_{\Lambda}^{+} \sim_{\Lambda}^{0}\right\}$. This allows us to control the fuzzy preference $\Lambda$ by means of the properties of these five associated binary relations, all crisp.

Definition 21. The 5-tuple $\left(\succ_{\Lambda^{\prime}}^{+} \succ_{\Lambda^{\prime}}^{0} \succ_{\Lambda^{\prime}}^{-} \sim_{\Lambda^{\prime}}^{+} \sim_{\Lambda}^{0}\right)$ is called the crisp spectrum of the fuzzy preference $\Lambda$.

We now introduce the abstract concept of a pseudo-fuzzy preference.

Definition 22. A pseudo-fuzzy preference $\Phi$ over a universe $U$ is a 5-tuple of (crisp) binary relations $\left(\succ_{1}, \succ_{2}\right.$ $\left., \succ_{3}, \sim_{1}, \sim_{2}\right)$ on $U$ such that there exists a total preorder $\succsim$ on $U$, whose asymmetric part is $\succ$ and its symmetric part is $\sim$, such that $\left\{\succ_{1}, \succ_{2} \succ_{3}\right\}$ is a decomposition of $\succ$ (i.e., $\succ=\succ_{1} \sqcup \succ_{2} \sqcup \succ_{3}$ ) and, in addition, $\left\{\sim_{1}, \sim_{2}\right\}$ is a decomposition of $\sim\left(\right.$ i.e., $\left.\sim=\sim_{1} \sqcup \sim_{2}\right)$. The relation $\succ($ respectively, $\sim)$ is said to be the asymmetric (respectively, the symmetric) part of the given pseudo-fuzzy preference. Henceforward, the set of all pseudo-fuzzy preferences over a set $U$ is denoted by $\pi_{U}$. (The symbol $\sqcup$ stands here for disjoint union. Thus, if $C=A \sqcup B$ we mean that $C=A \cup B$ and, in addition, $\varnothing=A \cap B$ ).

Proposition 6. Given a fuzzy preference $\Lambda=(\mathcal{R}, \mathcal{P}, \mathcal{I})$ on a universe $U$, the tuple $\left(\succ_{\Lambda^{\prime}}^{+} \succ_{\Lambda^{\prime}}^{0} \succ_{\Lambda^{\prime}}^{-} \sim_{\Lambda^{\prime}}^{+} \sim_{\Lambda}^{0}\right)$ is indeed a pseudo-fuzzy preference over $U$. 
Proof. Define $\succ$ as $\succ_{\Lambda}^{+} \sqcup \succ_{\Lambda}^{0} \sqcup \succ_{\Lambda}^{-}$and $\sim$ as $\sim_{\Lambda}^{+} \sqcup \sim_{\Lambda}^{0}$. We will see that these relations $\succ$ and $\sim$ are, respectively, the asymmetric and the symmetric part of a total preorder $\succsim$. To see this, observe that, by its own definition $\succ$ is asymmetric while $\sim$ is symmetric. Moreover, its intersection is empty. Furthermore, it is straightforward to see that $\succsim$ defined as $\succ \sqcup \sim$ is total. In particular, it is reflexive. Finally, taking into account that, for every $x, y \in U, x \succsim y$ is equivalent to $\mathcal{R}(x, y) \geq \mathcal{R}(y, x)$, and using the transitivity of the fuzzy preference $\Lambda$, we may also conclude that $\succsim$ is a transitive (crisp) binary relation on $U$. Therefore $\succsim$ is actually a total preorder.

Remark 5. If $\Lambda$ is indeed crisp, we get a crisp spectrum in which $\succ_{\Lambda^{\prime}}^{0} \succ_{\Lambda}^{-}$and $\sim_{\Lambda}^{0}$ are empty. So $\succ$ $($ respectively, $\sim)$ coincides with $\succ^{+}($respectively, with $\sim)$, and $\succsim($ respectively, $\succ, \sim)$ is $\mathcal{R}($ respectively, $\mathcal{P}, \mathcal{I})$.

Leaning on the concept of equivalence $\approx$ given in Definition 12 , we define now a new equivalence on the set $\mathcal{P}_{U}$ of fuzzy preferences on the universe $U$.

Definition 23. We call pairwise similarity to the equivalence relation $\equiv$ defined as follows on the set $\mathcal{P}_{U}$ of fuzzy preferences on a universe $U$ : Given $\Lambda, \Lambda^{\prime} \in \mathcal{P}_{U}$ we declare that $\Lambda$ is pairwise similar to $\Lambda^{\prime}$, and denote it by $\Lambda \equiv \Lambda^{\prime}$ if and only if $\Lambda_{\rceil\{x, y\}} \approx \Lambda_{\rceil\{x, y\}}^{\prime}$ holds true for any pair $(x, y) \in U \times U$.

The pairwise similarity just introduced in Definition 23 helps us to handle fuzzy preferences by means of their crisp spectra, as stated in the next result.

Theorem 4. There exists an injection from the quotient set of equivalence classes $\overline{\mathcal{P}_{U}}$ that the equivalence relation $\equiv$ induces on $\mathcal{P}_{U}$ into the set $\pi_{U}$ of the pseudo-fuzzy preferences on the universe $U$.

Proof. First consider the function $\bar{\theta}: \mathcal{P}_{U} \rightarrow \pi_{U}$ defined as $\bar{\theta}(\Lambda)=\left(\succ_{\Lambda^{\prime}}^{+} \succ_{\Lambda^{\prime}}^{0} \succ_{\Lambda^{\prime}}^{-} \sim_{\Lambda^{\prime}}^{+} \sim_{\Lambda}^{0}\right)$. One may easily check that given two fuzzy preferences $\Lambda, \Lambda^{\prime} \in \mathcal{P}_{U}$ it holds true that $\bar{\theta}(\Lambda)=\bar{\theta}\left(\Lambda^{\prime}\right) \Leftrightarrow \Lambda \equiv \Lambda^{\prime}$, so the map $\theta$ from $\overline{\mathcal{P}_{U}}$ into $\pi_{U}$ given by $\theta([\Lambda])=\bar{\theta}(\Lambda)$, is actually an embedding.

Definition 24. The range $D=\theta\left(\overline{\mathcal{P}_{U}}\right)$ is said to be the core of the set of pseudo-fuzzy preferences.

Remark 6. The pairwise similarity has been motivated by the property of independence of irrelevant alternatives when imposed to aggregation functions for fuzzy preferences Thus, given a n-aggregation rule $f: \mathcal{P}_{U}^{n} \rightarrow \mathcal{P}_{U}$ that satisfies the property of independence of irrelevant alternatives, we may straightforwardly see that it is compatible with the equivalence relation $\equiv$, in the following sense: given any two profiles $\left(\Lambda_{i}\right),\left(\Lambda_{i}^{\prime}\right) \in \mathcal{P}_{U}^{n}$, whose components are equivalent as regards $\equiv$ (i.e., $\Lambda_{i} \equiv \Lambda_{i}^{\prime}$ holds true for all $\left.i \in N\right)$, then their images through $f$ are also equivalent, (i.e., $f\left(\left(\Lambda_{i}\right)\right) \equiv f\left(\left(\Lambda_{i}^{\prime}\right)\right)$ also holds).

The fact that aggregation rules behave well with respect to $\equiv$ allows us to work directly on the quotient space of $\mathcal{P}_{U}$ through $\equiv$. Thus, given a n aggregation rule $f: \mathcal{P}_{U}^{n} \rightarrow \mathcal{P}_{U}$ satisfying independence of irrelevant alternatives, we may directly consider the new map $[f]: \overline{\mathcal{P}}_{U}{ }^{n} \rightarrow \overline{\mathcal{P}_{U}}$ as $[f]\left(\left(\left[\Lambda_{i}\right]\right)\right)=\left[f\left(\left(\Lambda_{i}\right)\right)\right]$. (Here $\overline{\mathcal{P}_{U}}$ denotes the quotient set of $\mathcal{P}_{U}$ through $\equiv$ ).

\subsection{Towards a Fuzzy Arrovian Impossibility Theorem}

The strategy to reach an Arrovian results in this context will consist of, first, considering the crisp spectrum of fuzzy preferences, and then proving that the impossibility of finding a rule à la Arrow to fuse those spectra (of crisp preferences) provokes the impossibility of aggregating in an Arrovian context the fuzzy preferences considered beforehand. To do so we introduce a new Arrovian model, in this case to deal with pseudo-fuzzy preferences on a universe $U$.

Definition 25. Let $E \subseteq \pi_{U}$ be a nonempty subset of the set of pseudo-fuzzy preferences on a universe $U$. Let $h: E^{n} \rightarrow \pi_{U}$ be a map, that we call pseudo-fuzzy aggregation rule. It is said that $h$ satisfies: 
(i) the property of independence of irrelevant alternatives if given any pair of profiles $\left(p_{i}\right),\left(p_{i}^{\prime}\right) \in E^{n}$ and $x, y \in U$ such that $p_{i\rceil\{x, y\}}=p_{i\rceil\{x, y\}}^{\prime}$ holds true for every $i \in N=\{1, \ldots, n\}$, then $h\left(\left(p_{i}\right)\right)_{\rceil\{x, y\}}=$ $h\left(\left(p_{i}^{\prime}\right)\right)_{1}\{x, y\}$,

(ii) the property of unanimity if given any $\left(p_{i}\right) \in E^{n}$ whose associated asymmetric parts are $\left(\succ_{i}\right)$, and $x, y \in$ $U$ such that $x \succ_{i} y$ holds true for every $i \in N=\{1, \ldots, n\}$, then $x \succ y$ also holds, (with $\succ$ being the asymmetric part of $\left.h\left(\left(p_{i}\right)\right)\right)$,

(iii) the property of dictatorship if there exist $k \in N=\{1, \ldots, n\}$ such that for every $\left(p_{i}\right) \in E^{n}$ and $x, y \in U$, it holds true that if $\succ_{k}$ stands for the asymmetric part of $p_{k}$, then $x \succ_{k}$ implies that $x \succ y$ (with $\succ$ denoting the asymmetric part of $\left.h\left(\left(p_{i}\right)\right)\right)$.

Let us introduce now a technique to interpret fuzzy aggregation rules through suitable associated pseudo-fuzzy aggregation rules. This leans on Theorem 4. Thus, let $\theta$ stand for the injection from the quotient set of equivalence classes $\overline{\mathcal{P}_{U}}$ that the equivalence relation $\equiv$ induces on $\mathcal{P}_{U}$ into the set $\pi_{U}$ of the pseudo-fuzzy preferences on the universe $U$, as stated in Theorem 4 . This injection gives rise to an inverse mapping $\theta^{-1}$ whose domain is $D \subseteq \pi_{U}$, namely the image of $\overline{\mathcal{P}}_{U}$ through $\theta$, also known as the core of the set of pseudo-fuzzy preferences. Let $f: \mathcal{P}_{U}^{n} \rightarrow \mathcal{P}_{U}$ be a $n$ aggregation rule satisfying independence of irrelevant alternatives. Let $[f]: \overline{\mathcal{P}_{U}}{ }^{n} \rightarrow \overline{\mathcal{P}_{U}}$ be defined as $[f]\left(\left(\left[\Lambda_{i}\right]\right)\right)=\left[f\left(\left(\Lambda_{i}\right)\right)\right]$. Now, define $\hat{f}: D^{n} \subseteq\left(\pi_{U}\right)^{n} \rightarrow \pi_{U}$ as $\hat{f}=\theta \circ[f] \circ\left(\left(\theta^{-1}\right)^{n}\right)$.

Definition 26. Given a n aggregation rule $f: \mathcal{P}_{U}^{n} \rightarrow \mathcal{P}_{U}$ that satisfies the property of independence of irrelevant alternatives, the corresponding map $\hat{f}: D^{n} \subseteq\left(\pi_{U}\right)^{n} \rightarrow \pi_{U}$ is said to be the crisp discretization of $f$.

Now we may already furnish some relationship between fuzzy aggregation rules and their corresponding crisp discretizations.

Proposition 7. Let $f: \mathcal{P}_{U}^{n} \rightarrow \mathcal{P}_{U}$ be a fuzzy n aggregation rule on the universe $U$. Suppose that $f$ satisfies the property of independence of irrelevant alternatives. Then its crisp discretization $\hat{f}$ also satisfies it. In addition, $f$ is Paretian (respectively, dictatorial) if and only if $\hat{f}$ satisfies unanimity (respectively, dictatorship).

Proof. Let $D \subseteq \pi_{U}$ be the core of pseudo-fuzzy preferences, namely the image of $\overline{\mathcal{P}_{U}}$ through $\theta$. Let $x, y \in U$. Suppose that $\left(p_{i}\right),\left(p_{i}^{\prime}\right) \in D^{n}$ are two profiles such that $p_{i\rceil\{x, y\}}=p_{i\rceil\{x, y\}}^{\prime}$ holds true for every $i \in N=\{1, \ldots, n\}$. Consider two profiles $\left(\Lambda_{i}\right),\left(\Lambda_{i}^{\prime}\right) \in \mathcal{P}_{U}^{n}$ with $\theta\left(\left[\Lambda_{i}\right]\right)=p_{i}$ and $\theta\left(\left[\Lambda_{i}^{\prime}\right]\right)=p_{i}^{\prime}$ such that $\Lambda_{i\rceil\{x, y\}} \approx \Lambda_{i\rceil\{x, y\}}^{\prime}$ holds true for any $1 \leq i \leq n$. Using the property of independence of irrelevant alternatives it follows that $f\left(\left(\Lambda_{i}\right)\right)_{\rceil\{x, y\}} \approx f\left(\left(\Lambda_{i}^{\prime}\right)\right)_{\rceil\{x, y\}}$. Hence $\hat{f}\left(\left(p_{i}\right)\right)_{\rceil\{x, y\}}=\theta\left(\left[f\left(\left(\Lambda_{i}\right)\right)\right]\right)_{\rceil\{x, y\}}=$ $\theta\left(\left[f\left(\left(\Lambda_{i}^{\prime}\right)\right)\right]\right)_{\rceil\{x, y\}}=\hat{f}\left(\left(p_{i}^{\prime}\right)\right)_{\rceil\{x, y\}}$.

If $\succ_{i}$ is the asymmetric part of $p_{i}$ and $x \succ_{i} y(1 \leq i \leq n)$, then there exist a profile $\left(\Lambda_{i}\right) \in \mathcal{P}_{U}^{n}$ with $\theta\left(\left[\Lambda_{i}\right]\right)=p_{i}$, and such that $\mathcal{R}_{i}(x, y)>\mathcal{R}_{i}(y, x)$, so $\mathcal{P}_{i}(x, y)>0$. Thus, by the Paretian property it follows that $\mathcal{P}_{f}(x, y)>0$. We may conclude that $x \succ y$, where $\succ$ denotes the asymmetric part of $\hat{f}\left(\left(p_{i}\right)\right)$, because this is equivalent to say that $\mathcal{R}_{f}\left(\left(\Lambda_{i}\right)\right)(x, y)>\mathcal{R}_{f}\left(\left(\Lambda_{i}\right)\right)(y, x)$.

As in Definition 3 (respectively, Theorem 3), denote by $\mathcal{O}_{U}$ (respectively, by $\mathcal{O}$ ) the set of all total preorders (respectively, of the asymmetric part of total preorders) defined on the universe $U$.

Definition 27. A decomposition rule for total preorders is a function $i: \mathcal{O}_{U} \rightarrow \pi_{U}$ such that the symmetric (respectively, the asymmetric) part of $\succsim \in \mathcal{O}_{U}$ and the corresponding symmetric (respectively asymmetric) part associated to $i(\succsim) \in \pi_{U}$ coincide. In other words, if $i(\succsim)=\left(\succ_{1}, \succ_{2}, \succ_{3}, \sim_{1}, \sim_{2}\right)$ then it holds true that $\succ=\succ_{1} \sqcup \succ_{2} \sqcup \succ_{3}$ and $\sim=\sim_{1} \sqcup \sim_{2}$. Furthermore, a decomposition rule $i$ is said to be compatible as regards the property of independence of irrelevant alternatives if for every pair $\succsim_{a}, \succsim_{b} \in \mathcal{O}_{U}$ and any two elements $x, y \in U$, it holds true that if $\succsim_{a\rceil\{x, y\}}=\succsim_{b\urcorner\{x, y\}}$, then $i\left(\succsim_{a}\right)_{\rceil\{x, y\}}=i\left(\succsim_{b}\right)_{\rceil\{x, y\}}$. 
Definition 28. Let $\mathcal{O}$ be the set of asymmetric parts of total preorders on the universe $U$. For any element $\succ$ that belongs to $\mathcal{O}$, there is a unique total preorder $\succsim$ on $U$ whose asymmetric part is $\succ$. In fact, for any $x, y \in U$ we have that $x \succsim y$ holds if and only if $y \succ x$ does not hold. The map $r: \mathcal{O} \rightarrow \mathcal{O}_{U}$ given by $r(\succ)=\succsim(\succ \in \mathcal{O})$ is said to be the completion of the asymmetric parts of total preorders.

Definition 29. The canonical projection $p: \pi_{U} \rightarrow \mathcal{O}$ is the function which assigns the asymmetric part of its associated preorder to each pseudo-fuzzy preference on the universe $U$.

Definition 30. Consider a nonempty domain $E \subseteq \pi_{U}$ and a pseudo-fuzzy aggregation rule $H: E^{n} \rightarrow \pi_{U}$, as well as a n-tuple of decomposition rules for total preorders, $\mathcal{D}=\left(i_{j}\right)_{j \in\{1, \ldots, n\}}$ satisfying that $i_{j}\left(\mathcal{O}_{U}\right) \subseteq$ $E(1 \leq j \leq n)$. The condensation of a pseudo-fuzzy aggregation rule $H \in \pi_{U}$ is now defined as follows: $H_{\mathcal{D}}=p \circ H \circ i_{\mathcal{D}}: \mathcal{O}^{n} \rightarrow \mathcal{O}$, where $i_{\mathcal{D}}=\left(i_{1} \circ r\right) \times \cdots \times\left(i_{n} \circ r\right)$. If, in addition, each $i_{j}$ is compatible with the independence of irrelevant alternatives, we will also say, as in Definition 27 , that $\mathcal{D}$ is compatible as regards the property of independence of irrelevant alternatives.

Proposition 8. Given a pseudo-fuzzy aggregation rule $H: E^{n} \rightarrow \pi_{U}$ and a n-tuple of decomposition rules $\mathcal{D}=\left(i_{j}\right)_{j \in\{1, \ldots, n\}}$ with $i_{j}\left(\mathcal{O}_{U}\right) \subseteq E(1 \leq j \leq n)$, if $H$ is unanimous, then $H_{\mathcal{D}}$ is also unanimous. Moreover, if $H$ satisfies the property of independence of irrelevant alternatives and $\mathcal{D}$ is compatible as regards that property, then $H_{\mathcal{D}}$ also satisfies independence of irrelevant alternatives.

Proof. Let $\left(\succsim_{i}\right),\left(\succsim_{i}^{\prime}\right) \in \mathcal{O}_{U}^{n}$ be two profiles, and $x, y$ two elements in the universe $U$. Let us prove first that $H_{\mathcal{D}}$ is unanimous: To do so, suppose that $x \succ_{i} y$ holds true for all $i \in\{1, \ldots, n\}$. Notice that $\succ_{i}$ is also the asymmetric part of $i_{i}\left(\succsim_{i}\right) \in \pi_{U}$. Since $H$ satisfies unanimity, the asymmetric part $\succ$ of $H\left(\left(i_{i}\left(\succsim_{i}\right)\right)\right)$ accomplishes that $x \succ y$. Hence $H_{\mathcal{D}}$ is also unanimous because $\succ$ is the asymmetric part of $H_{\mathcal{D}}\left(\left(\succ_{i}\right)\right)=p\left(H\left(\left(i_{i}\left(\succsim_{i}\right)\right)\right)\right)$. Assume now that $\succsim_{i\rceil\{x, y\}}=\succsim_{i\rceil\{x, y\}}^{\prime}$ holds true for any $1 \leq i \leq n$. From this assumption, and because of the hypothesis of compatibility with the property of independence of irrelevant alternatives, it follows that $i_{i}\left(\succsim_{i}\right)_{\rceil\{x, y\}}=i_{i}\left(\succsim_{i}^{\prime}\right)_{\rceil\{x, y\}}$ holds true for all $i \in\{1, \ldots, n\}$. Finally, once more by the independence of irrelevant alternatives, we conclude that $H\left(\left(i_{i}\left(\succsim_{i}\right)\right)\right)_{\rceil\{x, y\}}=H\left(\left(i_{i}\left(\succsim_{i}^{\prime}\right)\right)\right)_{\rceil\{x, y\}}$, so that $H_{\mathcal{D}}\left(\left(\succ_{i}\right)\right)_{\rceil\{x, y\}}=H_{\mathcal{D}}\left(\left(\succ_{i}\right)\right)_{\rceil\{x, y\}}$.

Proposition 9. Let $H$ be a pseudo-fuzzy aggregation rule on a universe $U$. Assume that $H$ satisfies unanimity and independence of irrelevant alternatives. Then, for any $n$-tuple of decomposition rules $\mathcal{D}=\left(i_{j}\right)_{j \in\{1, \ldots, n\}}$, compatible as regards the property of independence of irrelevant alternatives, and such that $i_{j}\left(\mathcal{O}_{U}\right) \subseteq E(1 \leq$ $j \leq n$ ), it holds true that $H_{\mathcal{D}}$ is a dictatorial (crisp) aggregation function. Besides, all the condensation maps $H_{\mathcal{D}}$ have the same dictator.

Proof. First of all, notice that by Proposition 8 , for each $\mathcal{D}$ we have that $H_{\mathcal{D}}: \mathcal{O}^{n} \rightarrow \mathcal{O}$ satisfies the hypotheses of the statement of Theorem 3. Hence $H_{\mathcal{D}}$ is indeed dictatorial. Denote its dictator by $\operatorname{di}\left(H_{\mathcal{D}}\right)$. We will prove in two steps that all the condensation maps $H_{\mathcal{D}}$ have the same dictator. To start with, we will prove it for two decompositions $\mathcal{D}$ and $\mathcal{D}^{\prime}$ such that there exist two alternatives $v, w \in U$ such that for all $i \in\{1, \ldots, n\}$ and any $\succ \in \mathcal{O}$ it holds true that $i_{i} \circ r(\succ)_{\rceil\{v, w\}}=i_{i}^{\prime} \circ r(\succ)_{\rceil\{v, w\}}$. Using independence of irrelevant alternatives we get that $\left.H_{\mathcal{D}}\left(\left(\succ_{i}\right)\right)_{\rceil\{v, w\}}=H_{\mathcal{D}^{\prime}}\left(\succ_{i}\right)\right)_{\rceil\{v, w\}}$ for all profiles $\left(\succ_{i}\right) \in \mathcal{O}^{n}$. If we consider a specific profile $\left(\succ_{i}\right) \in \mathcal{O}^{n}$ satisfying $w \succ_{i} v$ if $i \neq d i\left(H_{\mathcal{D}}\right)$ and $v \succ_{d i\left(H_{\mathcal{D}}\right)} w$, then it is clear that $\operatorname{di}\left(H_{\mathcal{D}^{\prime}}\right)=\operatorname{di}\left(H_{\mathcal{D}}\right)$ because $H_{\mathcal{D}^{\prime}}\left(\left(\succ_{i}\right)\right)$ strictly prefers $v$ to $w$. In the second step, given two arbitrary decompositions $\mathcal{D}$ and $\mathcal{D}^{\prime}$, we take a (fixed) couple $a, b \in U$ and define a third decomposition $\mathcal{D}^{\prime \prime}$ as follows: $i_{\mathcal{D}}^{\prime \prime}(\succsim)=q$ with $\left.\left.q\right\rceil\{a, b\}=i_{\mathcal{D}}(\succsim)\right\rceil\{a, b\}$ and $q_{\rceil\{x, y\}}=i_{\mathcal{D}^{\prime}}(\succsim)_{\rceil\{x, y\}}$ if $\{x, y\} \neq\{a, b\}$. Clearly $\mathcal{D}$ and $\mathcal{D}^{\prime \prime}$ satisfy the conditions of the case just studied over the pair $\{a, b\}$, whereas $\mathcal{D}^{\prime}$ and $\mathcal{D}^{\prime \prime}$ do the same over every pair different from $\{a, b\}$, so $\operatorname{di}\left(H_{\mathcal{D}}\right)=\operatorname{di}\left(H_{\mathcal{D}^{\prime \prime}}\right)=\operatorname{di}\left(H_{\mathcal{D}^{\prime}}\right)$. 
Remark 7. In the following Proposition 10, the core of pseudo-fuzzy preferences D is the domain over which we require the existence of decomposition rules. In such case, that is, whenever $E=D$, decomposition rules always exist, for example we can consider $i(\succsim)=\theta\left(\left(R_{\succsim}, P_{\succsim}, I_{\succsim}\right)\right)$, where $R_{\succsim}, P_{\succsim}$ and $I_{\succsim}$ are defined for all $x, y \in U$ as: $R_{\succsim}(x, y)=1$ if $x \succsim y$ and $R_{\succsim}(x, y)=0$ otherwise, $P_{\succsim}(x, y)=\tilde{1}$ if $x \succ y$ and $P_{\succsim}(x, y)=0$ otherwise, and $\tilde{I_{\succsim}}(x, y)=1$ if $x \sim y$ and $I_{\succsim} \widetilde{(x, y)}=0$ otherwise. The rule $i$ is a decomposition rule.

However, decomposition rules may fail to exist for a domain $E \subseteq \pi_{U}$ such that $E \neq D$. (In that case, obviously, Propositions 8 and 9 become trivial). For example, consider a domain $E_{1}$ containing a single pseudo-fuzzy preference, i.e., $E_{1}=\left\{\succ_{1}, \succ_{2}, \succ_{3}, \sim_{1}, \sim_{2}\right\} \subseteq \pi_{U}$. There is no decomposition rule $i$ satisfying $i\left(\mathcal{O}_{U}\right) \subseteq E$, because the asymmetric part of any total preorder from $\mathcal{O}_{U}$ should agree with the asymmetric part of $\left(\succ_{1}, \succ_{2}, \succ_{3}, \sim_{1}, \sim_{2}\right)$.

Proposition 10. Let $D \subseteq \pi_{U}$ be the core of pseudo-fuzzy preferences. Let $H: D^{n} \rightarrow \pi_{U}$ be a pseudo-fuzzy aggregation rule on the universe $U$. Assume that $H$ satisfies unanimity and independence of irrelevant alternatives. Then $H$ is dictatorial.

Proof. Consider an arbitrary decomposition $\mathcal{D}$, and define $k=d i\left(H_{\mathcal{I}}\right)$. Let us prove now that $k$ is also the dictator of $H$. Let $\left(p_{i}\right) \in D^{n}$ be a profile with asymmetric parts $\left(\succ_{i}\right)$. Suppose that there exist $x, y \in U$ with $x \succ_{k} y$. Let $\mathcal{D}^{\prime}$ be the decomposition defined as $\mathcal{D}^{\prime}=\left(i_{j}\right)_{j \in\{1, \ldots, n\}}$ with $i_{j}(\succsim)=p_{j}$ if $\succ=p\left(p_{j}\right)$ and $i_{j}(\succsim)=\theta\left(\left(R_{\succsim}, P_{\succsim}, I_{\succsim}\right)\right)$ if $\succ \neq p\left(p_{j}\right)$, where $R_{\succsim}, P_{\succsim}$ and $I_{\succsim}$ are defined as in Remark 7 . By Proposition 9 , the element $k$ is the dictator of $H_{\mathcal{D}^{\prime}}$, so $p \circ \tilde{H}\left(\left(\tilde{p}_{i}\right)\right) \in \tilde{\mathcal{O}}$ strictly prefers $x$ to $y$. Thus we may conclude that if $\succ$ is the asymmetric part of $H\left(\left(p_{i}\right)\right)$, then $x \succ y$ holds.

Finally, we will announce the main result of this section:

Theorem 5. Let $f:\left(\mathcal{P}_{U}\right)^{n} \rightarrow \mathcal{P}_{U}$ be a fuzzy aggregation rule satisfying independence of irrelevant alternatives as well as the Paretian property. Then $f$ is dictatorial.

Proof. By Proposition $7, f$ is dictatorial if and only if $\hat{f}$ is dictatorial. Besides, $\hat{f}$ satisfies the hypotheses of Proposition 10, so $\hat{f}$ is indeed dictatorial. Hence $f$ is dictatorial, too.

\section{Discussion}

In spite of similar results to the main Theorem 5 having already appeared in the literature (see e.g., [8]), the technique introduced in the present manuscript is new, as far as we now. This technique, based in the introduction of the so-called pseudo-fuzzy preferences (that are indeed suitable crisp binary relations) allows us to come back to the crisp setting to interpret and deduce key situations arising in the fuzzy approach. Among them, obviously, the most relevant one is a new impossibility theorem that tries to retrieve the spirit of the Arrovian models arising for crisp preferences. In addition, our definition of a fuzzy preference is a bit less restrictive that the usual ones encountered in this literature, since we use $\cup_{f}$-connectedness instead of completeness. At this stage, having in mind that there exist other fuzzy contexts where one may encounter possibility theorems of existence of aggregation rules à la Arrow (see e.g., $[7,8]$ ) one may wonder which is the scope of the new technique introduced here, and why it allows us to deal with fuzzy preferences by means of auxiliary crisp binary relations based on total preorders. In particular, we may ask ourselves about why here we reach an impossibility Arrovian result, whereas in other fuzzy contexts some possibility theorems are still available. An analysis in depth of these questions shows us that, on the one hand, the definitions of a fuzzy preference arising in those other alternative fuzzy contexts that gave rise to possibility results are different from the one introduced here in Definition 17. In fact, other kinds of transitivity are used there. The key that allows us to pass to control fuzzy preferences by means of crisp binary relations in our context is just the particular condition of independence of irrelevant alternatives that is used in the present manuscript, introduced in Definition 20. With this version of that crucial property, the new 
technique launched then, which allows us to fuse fuzzy preferences in terms of their crisp spectra, works well and gives rise to an Arrovian impossibility theorem in the fuzzy setting.

\section{Conclusions}

When the classical social choice model to aggregate individual preferences into a social one, introduced by K.J. Arrow in the 1950's, is extended to the fuzzy setting, both possibility and impossibility results may appear, as encountered in the existing specialized literature. To fuse individual fuzzy preferences one may consider some particular kinds of decompositions that, as analyzed in the present manuscript, allow us to handle the fuzzy preferences by means of a bunch of crisp of binary relations, all based in total preorders, that we name pseudo-fuzzy preferences. Thus, using the classical (crisp) Arrow's impossibility theorem, we get an impossibility theorem in the fuzzy approach. The technique introduced constitutes a novelty.

Author Contributions: All authors have equally contributed. All authors have read and agreed to the published version of the manuscript.

Funding: This work is partially supported by the research projects MTM2015-63608-P and TIN2016-77356-P (MINECO/ AEI-FEDER, UE).

Acknowledgments: Thanks are given to three anonymous referees for their valuable suggestions and comments on previous versions of the manuscript.

Conflicts of Interest: The authors declare no conflicts of interest.

\section{References}

1. Arrow, K.J. A difficulty in the concept of social welfare. J. Political Econ. 1950, 58, 328-346.

2. Arrow, K.J. Social Choice and Individual Values; Wiley: New York, NY, USA, 1951.

3. Arrow, K.J. Social Choice and Individual Values; 2nd ed.; Wiley: New York, NY, USA, 1963. (In this second edition some flaw arising in the original proofs of Arrow's impossibility problem given in [1,2] were mended and corrected. The mistakes in the former proofs had been pointed out by Julian Blau in [4]).

4. Blau, J.H. The existence of social welfare functions. Econometrica 1957, 25, 302-313.

5. Kelly, J.S. Arrow Impossibility Theorems; Academic Press: New York, NY, USA, 1978.

6. Kelly, J.S. Social Choice Theory. An Introduction; Springer: Berlin, Germany, 1988.

7. Dutta, B. Fuzzy preferences and social choice. Math. Soc. Sci. 1987, 13, 215-229.

8. Gibilisco, M.B.; Gowen, A.M.; Albert, K.E.; Mordeson, J.N.; Wierman, M.J.; Clark, T.D. Fuzzy Social Choice Theory. In Studies in Fuzziness and Soft Computing; Springer International Publishing: Cham, Switzerland, 2014; Volume 315.

9. Mordeson, J.N.; Gibilisco, M.B.; Clark, T.D. Independence of irrelevant alternatives and fuzzy Arrow's theorem. New Math. Nat. Comput. 2012, 8, 219-237.

10. Montero, F.J. The impact of fuzziness in social choice paradoxes. Soft Comput. 2008, 12, 177-182.

11. Barrett, C.R.; Pattanaik, P.K.; Salles, M. On the structure of fuzzy social welfare functions. Fuzzy Sets Syst. 1986, 19, 1-10.

12. Cholewa, W. Aggregation of fuzzy opinions: An axiomatic approach. Fuzzy Sets Syst. 1985, 17, 249-258.

13. Fung, L.W.; Fu, K.S. An Axiomatic Approach to Rational Decision Making in a Fuzzy Environment; Zadeh, L.A., Fu, K.S., Tanaka, K., Shimura, M., Eds.; Fuzzy Sets and Their Applications to Cognitive and Decission Processes; Academic Press: New York, NY, USA, 1975; pp. 227-256.

14. Montero, F.J. A note on Fung-Fu's theorem. Fuzzy Sets Syst. 1985, 17, 259-269.

15. Montero, F.J. Aggregation of fuzzy opinions in a non-homogeneous group. Fuzzy Sets Syst. 1988, 25, 15-20.

16. Billot, A. Aggregation of preferences: The fuzzy case. Theory Decis. 1991, 30, 51-93

17. Billot, A. Economic Theory of Fuzzy Equilibria: An Axiomatic Analysis; Lecture Notes in Economics and Mathematical Systems; Springer: Berlin, Germany, 1992.

18. Dasgupta, M.; Deb, R. An Impossibility Theorem with Fuzzy Preferences; de Swart, H., Ed.; Logic, Game Theory and Social Choice; Tilburg University Press: Tilburg, The Netherlands, 1999; pp. 482-490.

19. Duddy, C.; Piggins, A. On some oligarchy results when social preference is fuzzy. Soc. Choice Welf. 2018, $51,717-735$. 
20. Fono, L.A.; Andjiga, N.G. Fuzzy strict preference and social choice. Fuzzy Sets Syst. 2005, 155, 372-389.

21. Fono, L.A.; Donfack-Kommogne, V.; Andjiga, N.G. Fuzzy Arrow-type results without the pareto principle based on fuzzy pre-orders. Fuzzy Sets Syst. 2009, 160, 2658-2672.

22. Richardson, G. The structure of fuzzy preferences: Social choice implications. Soc. Choice Welf. 1998, 15, 359-369.

23. Fishburn, P.C. Arrow's impossibility theorem: Concise proof and infinite voters. J. Econom. Theory 1970, 2, 103-106.

24. Ekeland, I. Éléments d' Économie Mathématique; Hermann: Paris, France, 1979.

25. Banerjee, A. Fuzzy preferences and Arrow-type problems in social choice. Soc. Choice Welf. 1994, 11, 121-130.

26. Ovchinnikov, S. Structure of fuzzy binary relations. Fuzzy Sets Syst. 1981, 6, 169-195.

27. Díaz, S.; De Baets, B.; Montes, S. Transitivity and negative transitivity in the fuzzy setting. Adv. Intell. Soft Comput. 2011, 107, 91-100.

28. Ovchivnnikov, S. Numerical representation of fuzzy transitive relations. Fuzzy Sets Syst. 2000, 126, $225-232$.

29. Agud, L.; Catalán, R.G.; Díaz, S.; Induráin, E.; Montes, S. Numerical representability of fuzzy total preorders. Int. J. Comput. Intell. Syst. 2012, 5, 996-1009.

30. Montes, I. Díaz, S.; Montes, S. On complete fuzzy preorders and their characterizations. Soft Comput. 2010, 15, 1999-2011.

31. Fono, L.A.; Andjiga, N.G. Utility function of fuzzy preferences on a countable set under max-star transitivity. Soc. Choice Welf. 2007, 28, 667-683.

32. Duddy, C.; Perote-Peña, J.; Piggins, A. Arrow's theorem and max-star transitivity. Soc. Choice Welf. 2011. $36,25-34$.

(C) 2020 by the authors. Licensee MDPI, Basel, Switzerland. This article is an open access article distributed under the terms and conditions of the Creative Commons Attribution (CC BY) license (http:/ / creativecommons.org/licenses/by/4.0/). 